\title{
Electronic Supplementary Information Pd-Catalyzed Hydroborylation of Alkynes: A Ligand Controlled Regioselectivity Switch for the Synthesis of $\alpha$ - or $\beta$-vinylboronates
}

\author{
Devi Prasan Ojha and Kandikere Ramaiah Prabhu* \\ Department of Organic Chemistry, Indian Institute of Science, \\ Bangalore560 012, Karnataka, India
}

\section{Table of Contents}

General Information

General procedure for the synthesis of IPr-Hcl

SI-2

General Procedures for $\alpha$-borylation of terminal alkynes

General Procedures for $\beta$-borylation of terminal alkynes

Regioselective borylation of hex-5-ynenitrile using MeOD

SI-3

Control experiments with different oxidizing agents for borylation

SI-3

Competitive experiments

Characterization

SI-4

References

SI-9

NMR Spectra

SI-10

nOe Exoeriment $\left(\mathbf{2} \mathbf{f}^{\prime}\right)$

SI-46 


\section{General Information}

All solvents were distilled under nitrogen atmosphere prior to use. 1, 4-Dioxane, toluene were dried over Na. Unless otherwise noted, commercially available chemicals were distilled and degassed before use. Palladium acetate (II) trimer (CAS:3375-31-3) was used for the reaction, For chromatography, 200-300 mesh silica gel was employed. ${ }^{1} \mathrm{H}$ spectra were recorded at 400 $\mathrm{MHz}$ and $500 \mathrm{MHz}$ and ${ }^{13} \mathrm{C} \mathrm{NMR}$ spectra were recorded at $100 \mathrm{MHz}$ and $125 \mathrm{MHz}$ spectrometer. Chemical shifts $\left({ }^{1} \mathrm{H}\right)$ are reported in ppm using tetramethylsilane as internal standard when $\mathrm{CDCl}_{3}$ was used as solvent. For ${ }^{13} \mathrm{C} \mathrm{NMR}$, the triplet of $\mathrm{CDCl}_{3}$ (the center peak at $\delta 77.00)$ is used as reference. IR spectra were recorded using Perkin Elmer FT-IR instrument. Mass spectra (EI) were recorded using Shimadzu QP-2010 Ultra and High resolution mass spectra (HRMS) were recorded on Q-TOF (Micromass) spectrometer.

\section{General procedure for the synthesis of IPr-Hcl ${ }^{1}$}

A dilute solution of $\mathrm{HCl}(69 \mathrm{~mL}$ of $4 \mathrm{M}$ solution in dioxane) was added into a well-stirred solution of diazabutadiene ((1E,2E)-N1,N2-bis(2,6-diisopropylphenyl)ethane-1,2-diimine) (3.76 $\mathrm{g}, 10 \mathrm{mmol})$ in $37 \mathrm{~mL}$ of ethyl acetate $(0.5 \mathrm{M})$, followed by addition of paraformaldehyde $(6.1 \mathrm{~g}$, $0.2 \mathrm{~mol})$. The reaction mixture was stirred at room temperature till the reaction mixture becomes homogeneous $(\sim 1 \mathrm{~h})$. (Paraformaldehyde solution should be added to the solution of diazabutadiene to maintain a gentle heating without reaching reflux of the solvent $(1 \mathrm{~h})$ ). The reaction mixture was stirred overnight at room temperature while the yellow colored reaction mixture turns to brown. The precipitate formed during the reaction was filtered and washed with ethyl acetate $(3 \times 100 \mathrm{~mL})$. After drying of the precipitate under vacuum $(16 \mathrm{~h})$, the product was obtained as an off-white solid. The solid was dissolved in a saturated aqueous solution of $\mathrm{NaHCO}_{3}$ and transferred into a separating funnel, washed with ethyl acetate $(3 \times 100 \mathrm{~mL})$, followed $\mathrm{CH}_{2} \mathrm{Cl}_{2}(3 \times 100 \mathrm{~mL})$. The combined organic phase was dried over $\mathrm{MgSO}_{4}(20-30 \mathrm{~g}$ and manually stirred for $1 \mathrm{~min}$ ), filtered and concentrated under vacuum with a rotary evaporator (pressure 580 mbar with a water bath temperature at $40{ }^{\circ} \mathrm{C}$ ). Recrystallized using a mixture of ethyl acetate and $\mathrm{CH}_{2} \mathrm{Cl}_{2}(450 \mathrm{~mL}, 8: 1)$. Filter the white precipitate and wash it three times with ethyl acetate $(3 \times 50 \mathrm{ml})$. Dry the product under vacuum.

\section{General Procedures for $\alpha$-borylation of terminal alkynes}

To an oven-dried Teflon capped vial equipped with a magnetic stirring bar were added sequentially bromobenzene (187 mg, $1 \mathrm{mmol}$ ), bis(pinacolato)diboron ( $278 \mathrm{mg}, 1.1 \mathrm{mmol}$ ), $\mathrm{PCy}_{3}$ $(28 \mathrm{mg}, 0.10 \mathrm{mmol}),\left[\mathrm{Pd}(\mathrm{OAc})_{2}\right]_{3}(4.0 \mathrm{mg}, 0.05 \mathrm{mmol})$, alkyne $(1 \mathrm{mmol})$, toluene $(2 \mathrm{~mL})$ and trifluoroethanol $(2 \mathrm{mmol})$ under argon. The mixture was stirred at $80{ }^{\circ} \mathrm{C}$ for $12 \mathrm{~h}$ (monitored by TLC). Upon completion of the reaction, the resulting mixture was filtered through a short column of silica gel (eluted with $\mathrm{Et}_{2} \mathrm{O}, 20 \mathrm{~mL} \times 3$ ) and concentrated. This crude mixture was further purified on a silica gel column chromatography to obtain pure product.

\section{General Procedures for $\boldsymbol{\beta}$-borylation of terminal alkynes}

To an oven-dried Teflon capped vial equipped with a magnetic stirring bar were added sequentially bromobenzene (187 mg, $1 \mathrm{mmol}$ ), bis(pinacolato)diboron ( $278 \mathrm{mg}, 1.1 \mathrm{mmol})$, IPr$\mathrm{HCl}$ (5 mmol\%), ${ }^{t} \mathrm{BuOK}(11 \mathrm{~mol} \%),\left[\mathrm{Pd}(\mathrm{OAc})_{2}\right]_{3}(4.0 \mathrm{mg}, 0.025 \mathrm{mmol})$, alkyne $(1 \mathrm{mmol})$, toluene $(2 \mathrm{~mL})$ and trifluoroethanol $(2 \mathrm{mmol})$ under argon. The mixture was stirred at $80{ }^{\circ} \mathrm{C}$ for $12 \mathrm{~h}$ (monitored by TLC). Upon completion of the reaction, the resulting mixture was filtered through a short column of silica gel (eluted with $\mathrm{Et}_{2} \mathrm{O}, 20 \mathrm{~mL} \times 3$ ) and concentrated. This crude mixture was further purified on a silica gel column chromatography to obtain pure product. 
$\underline{\text { Regioselective borylation of hex-5-ynenitrile using MeOD }}$

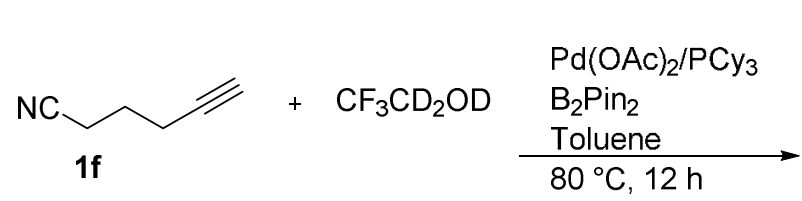

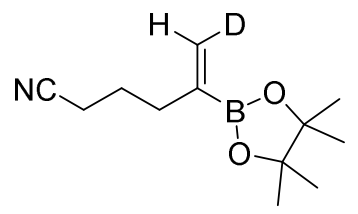

$40 \%$ Deuterated<smiles>[2H]C=C(CCCC#N)B1OC(C)(C)C(C)(C)O1</smiles>

$60 \%$ - Deuterated

Following the general procedure described above, (E)-5-(4,4,5,5-tetramethyl-1,3,2-dioxaborolan2-yl)hex-5-enenitrile-6-d was obtained as a colourless liquid, $62.4 \mathrm{mg}\left(71 \%, 60 \%{ }^{2} \mathrm{H}\right.$ insertion). ${ }^{1} \mathrm{H}$ NMR $\left(\mathrm{CDCl}_{3}, 300 \mathrm{MHz}\right): \delta 1.26(\mathrm{~s}, 12 \mathrm{H}), 1.77-1.84(\mathrm{~m}, 2 \mathrm{H}), 2.14(\mathrm{q}, J=7.2 \mathrm{~Hz}, 4 \mathrm{H}), 5.66$ $(\mathrm{s}, 1 \mathrm{H}), 5.86(\mathrm{~d}, J=2.8 \mathrm{~Hz}, 1 \mathrm{H},) ;{ }^{13} \mathrm{C} \mathrm{NMR}\left(\mathrm{CDCl}_{3}\right): \delta 16.4,24.7,24.98,34.3,83.5,131.1$. The stereochemistry was confirmed by a $1 \mathrm{D}$ - nOe experiment of the non- $=$ deuterated product.

\section{Control experiments with different oxidizing agents for borylation}

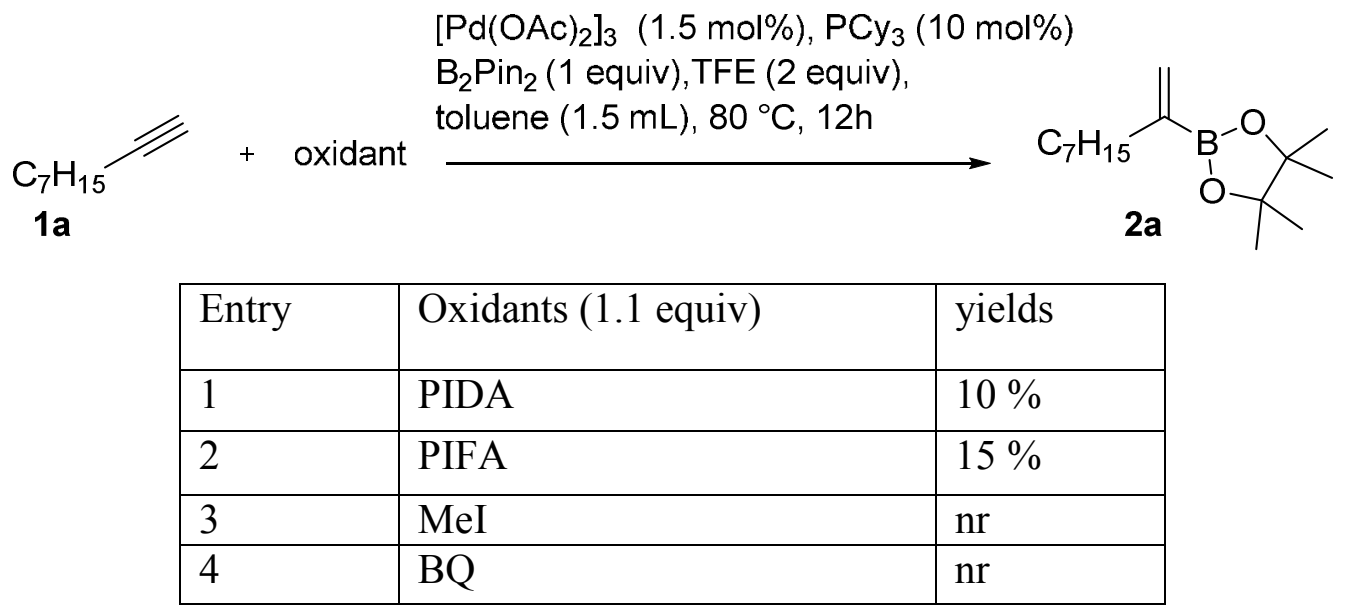

\section{Competitive experiments}

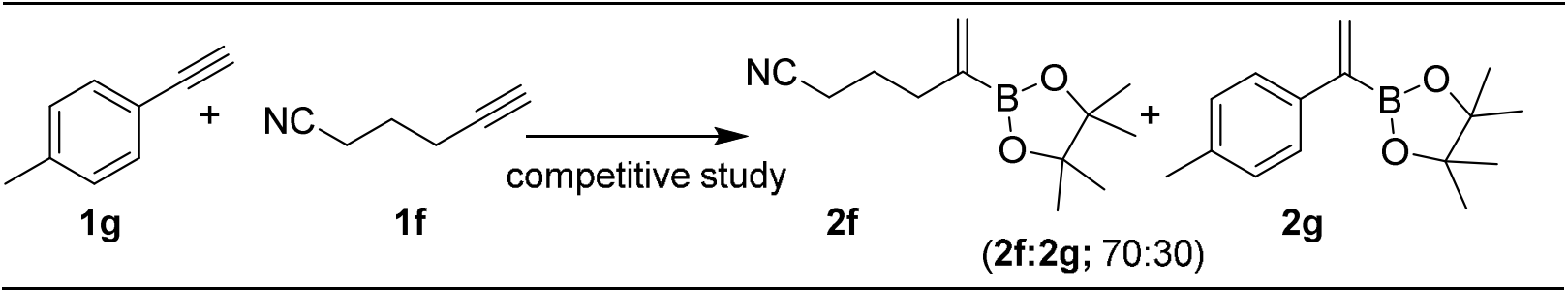

To an oven-dried Teflon capped vial equipped with a magnetic stirring bar were added sequentially bromobenzene (187 mg, $1 \mathrm{mmol}$ ), bis(pinacolato)diboron ( $278 \mathrm{mg}, 1.1 \mathrm{mmol}$ ), $\mathrm{PCy}_{3}$ $(28 \mathrm{mg}, 0.10 \mathrm{mmol}),\left[\mathrm{Pd}(\mathrm{OAc})_{2}\right]_{3}(4.0 \mathrm{mg}, 0.05 \mathrm{mmol})$, alkynes $\mathbf{1 g}(0.5 \mathrm{mmol})$ and $\mathbf{1 f}(0.5$ $\mathrm{mmol})$ were subjected for a competitive hydroborylation in toluene $(2 \mathrm{~mL})$ and trifluoroethanol ( $2 \mathrm{mmol}$ ) under argon. The mixture was stirred at $80^{\circ} \mathrm{C}$ for $12 \mathrm{~h}$ (monitored by TLC). Upon completion of the reaction, the resulting mixture was filtered through a short column of silica gel (eluted with $\mathrm{Et}_{2} \mathrm{O}, 20 \mathrm{~mL} \times 3$ ) and concentrated. This reaction resulted in distinguishable borylated products $\mathbf{2 f}$ and $\mathbf{2 g}$ in 70:30 ratios and the conversion was obtained by ${ }^{1} \mathrm{H}-\mathrm{NMR}$ spectra (see spectral section below). 


\section{Characterization}

\section{4,4,5,5-tetramethyl-2-(1-phenylvinyl)-1,3,2-dioxaborolane: ${ }^{1}$}

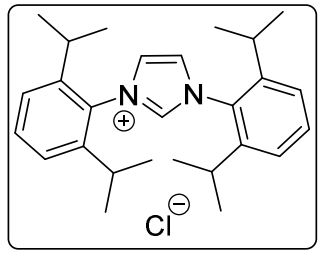

Purified by flash chromatography on silica gel using EtOAc: hexane (1:10) as eluent. $\mathrm{R}_{f}$ (hexane) $=0.5$. off-white solid; Yield: 41\%, $\left(1.74 \mathrm{~g}\right.$ ); IR (neat, $\left.\mathrm{cm}^{-1}\right): 3105,2989,1521,1461,1454$, 1168, 1065, 1038; ${ }^{1} \mathbf{H}$ NMR $\left(\mathrm{CDCl}_{3}, 400 \mathrm{MHz}\right): \delta 10.1(\mathrm{~s}, 1 \mathrm{H}), 8.13(\mathrm{~s}, 2 \mathrm{H}), 7.57(\mathrm{t}, J=7.6 \mathrm{~Hz}$, 2H), 7.35 (d, $J=8.0 \mathrm{~Hz}, 4 \mathrm{H}), 2.45$ (pent, $J=6.8 \mathrm{~Hz}, 4 \mathrm{H}), 1.29$ (d, $J=6.8 \mathrm{~Hz}, 12 \mathrm{H}), 1.24(\mathrm{~d}, J=$ $6.8 \mathrm{~Hz}, 12 \mathrm{H}) ;{ }^{13} \mathrm{C}$ NMR $\left(\mathrm{CDCl}_{3}\right): \delta 24.2,28.4,124.3,125.5,128.9,142.8$; HRMS (ESI) : calculated for $\mathrm{C}_{27} \mathrm{H}_{37} \mathrm{ClN}_{2}$ : 424.2645; Found: 424.2648. The physical data were identical to those previously reported data.

\section{4,4,5,5-tetramethyl-2-(non-1-en-2-yl)-1,3,2-dioxaborolane): ${ }^{2}$}

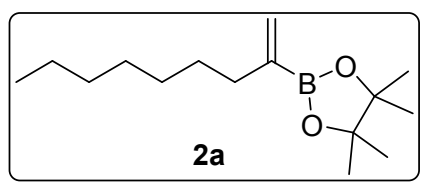

Purified by flash chromatography on silica gel using EtOAc : hexane (1:10) as eluent. $\mathrm{R}_{f}$ (hexane) $=0.5$. Colourless liquid; Yield: $85 \%$, (214 mg); IR (neat, $\mathrm{cm}^{-1}$ ): 3452, 2959, 1598, 1454, 1065; ${ }^{1} \mathbf{H}$ NMR $\left(\mathrm{CDCl}_{3}, 400 \mathrm{MHz}\right): \delta 0.87-0.89(\mathrm{~m}, 3 \mathrm{H}), 1.26(\mathrm{~s}, 20 \mathrm{H}), 1.39-1.42(\mathrm{~m}, 2 \mathrm{H})$, $2.14(\mathrm{t}, J=7.2 \mathrm{~Hz}, 2 \mathrm{H}), 5.58(\mathrm{~s}, 1 \mathrm{H}), 5.74(\mathrm{~d}, J=2.8 \mathrm{~Hz}, 1 \mathrm{H},) ;{ }^{13} \mathbf{C} \mathbf{N M R}\left(\mathrm{CDCl}_{3}\right): \delta$ 14.1, 22.6, 24.7, 24.8, 29.19, 29.2, 31.8, 35.3, 83.2, 128.6; MS (m/z) (rel. intensity): 252 (11), 237 (73), 153 (100). The physical data were identical to those previously reported data.

\section{4,4,5,5-tetramethyl-2-(oct-1-en-2-yl)-1,3,2-dioxaborolane): ${ }^{3}$}

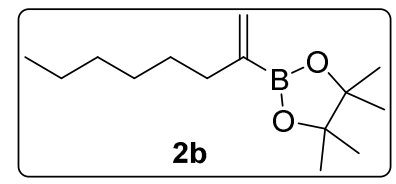

Purified by flash chromatography on silica gel using EtOAc : hexane (1:10) as eluent. $\mathrm{R}_{f}$ (hexane) $=0.5$. Colourless liquid; Yield: $73 \%,(173 \mathrm{mg})$; IR (neat, $\left.\mathrm{cm}^{-1}\right): 3467,2928,2617$, 1362, 1318; ${ }^{1} \mathbf{H}$ NMR $\left(\mathrm{CDCl}_{3}, 400 \mathrm{MHz}\right): \delta$ 0.87-0.89 (m, 3H), $1.26(\mathrm{~s}, 19 \mathrm{H}), 1.39-1.42(\mathrm{~m}, 2 \mathrm{H})$, $2.14(\mathrm{t}, J=7.2 \mathrm{~Hz}, 2 \mathrm{H}), 5.59(\mathrm{~s}, 1 \mathrm{H}), 5.75(\mathrm{~d}, J=2.8 \mathrm{~Hz}, 1 \mathrm{H},) ;{ }^{13} \mathbf{C}$ NMR $\left(\mathrm{CDCl}_{3}\right): \delta 14.1,22.6$, 24.7, 29.19, 29.2, 31.8, 35.3, 83.2, 128.6; MS (m/z) (rel. intensity): 238 (10), 223 (53), 153 (100). The physical data were identical to those previously reported data. 
2-(hept-1-en-2-yl)-4,4,5,5-tetramethyl-1,3,2-dioxaborolane: ${ }^{4}$

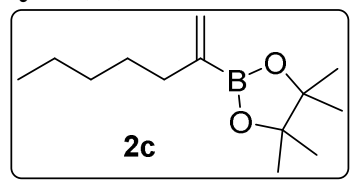

Purified by flash chromatography on silica gel using EtOAc : hexane (1:10) as eluent. $\mathrm{R}_{f}$ (hexane) $=0.5$. Colourless liquid; Yield: 69\%, (154 mg); IR (neat, $\left.\mathrm{cm}^{-1}\right): 3658,2974,2927$, 1638,$1462 ;{ }^{1} \mathbf{H}$ NMR $\left(\mathrm{CDCl}_{3}, 400 \mathrm{MHz}\right): \delta 5.75(\mathrm{~d}, J=2.8 \mathrm{~Hz}, 1 \mathrm{H}), 5.59(\mathrm{~s}, 1 \mathrm{H}), 2.13(\mathrm{t}, J=7.6$ $\mathrm{Hz}, 2 \mathrm{H}), 1.42-1.37(\mathrm{~m}, 2 \mathrm{H}), 1.26(\mathrm{~m}, 18 \mathrm{H}), 0.88-0.86(\mathrm{~m}, 3 \mathrm{H}) ;{ }^{13} \mathbf{C}$ NMR $\left(\mathrm{CDCl}_{3}\right): \delta 14.0,22.5$, 24.7, 24.8, 28.8, 31.4, 35.2, 83.2, 128.6; MS (m/z) (rel. intensity): 224 (11), 209 (53), 153 (100). The physical data were identical to those previously reported data.

\section{2-(hex-1-en-2-yl)-4,4,5,5-tetramethyl-1,3,2-dioxaborolane: ${ }^{3}$}

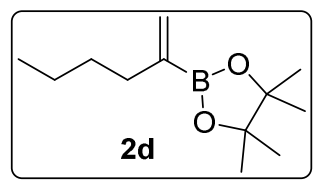

Purified by flash chromatography on silica gel using EtOAc : hexane $(1: 10)$ as eluent. $\mathrm{R}_{f}$ (hexane) $=0.5$. Colourless liquid; Yield: $65 \%$, (136 mg); IR (neat, $\left.\mathrm{cm}^{-1}\right): 3429,2989,1557$, 1479, 1444, 1168, 1015; ${ }^{1} \mathbf{H}$ NMR $\left(\mathrm{CDCl}_{3}, 400 \mathrm{MHz}\right): \delta 0.87-0.89(\mathrm{~m}, 3 \mathrm{H}), 1.26(\mathrm{~s}, 13 \mathrm{H}), 1.36-$ $1.43(\mathrm{~m}, 4 \mathrm{H}), 2.14(\mathrm{t}, J=7.2 \mathrm{~Hz}, 2 \mathrm{H}), 5.58(\mathrm{~s}, 1 \mathrm{H}), 5.75(\mathrm{~d}, J=2.8 \mathrm{~Hz}, 1 \mathrm{H}) ;{ }^{13} \mathbf{C}$ NMR (CDCl3): $\delta$ 14.1, 22.3, 24.7, 31.4, 35.0, 83.2, 128.5; MS (m/z) (rel. intensity): 210 (12), 195 (59), 153 (100). The physical data were identical to those previously reported data.

\section{4,4,5,5-tetramethyl-2-(pent-1-en-2-yl)-1,3,2-dioxaborolane: ${ }^{5}$}

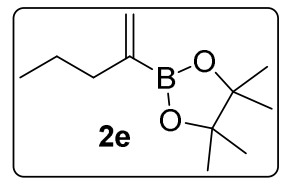

Purified by flash chromatography on silica gel using EtOAc : hexane (1:10) as eluent. $\mathrm{R}_{f}$ (hexane) $=0.5$. Colourless liquid; Yield: 55\%, (107 mg); IR (neat, $\left.\mathrm{cm}^{-1}\right): 3412,2939,1587$, $1478,1457,1169,1055,1028 ;{ }^{1} \mathbf{H}$ NMR $\left(\mathrm{CDCl}_{3}, 400 \mathrm{MHz}\right): \delta 0.87-0.89(\mathrm{~m}, 3 \mathrm{H}), 1.26(\mathrm{~s}, 12 \mathrm{H})$, 1.41-1.46 (m, 2H), $2.12(\mathrm{t}, J=7.2 \mathrm{~Hz}, 2 \mathrm{H}), 5.59(\mathrm{~s}, 1 \mathrm{H}), 5.76(\mathrm{~d}, J=2.8 \mathrm{~Hz}, 1 \mathrm{H}$,$) ; { }^{13} \mathbf{C}$ NMR $\left(\mathrm{CDCl}_{3}\right): \delta 13.7,22.3,24.7,37.5,83.2,128.89 ; \mathbf{M S}(\mathrm{m} / \mathrm{z})$ (rel. intensity): 196 (21), 181 (40), 139 (100). The physical data were identical to those previously reported data.

5-(4,4,5,5-tetramethyl-1,3,2-dioxaborolan-2-yl)hex-5-enenitrile: ${ }^{6}$

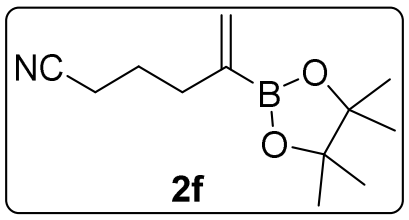

Purified by flash chromatography on silica gel using EtOAc : hexane (1:10) as eluent. $\mathrm{R}_{f}$ (hexane) $=0.5$. Colourless liquid; Yield: 89\%, (189 mg); IR (neat, $\left.\mathrm{cm}^{-1}\right): 3022,2269,1587$, 1068, 1065, 938; ${ }^{1} \mathbf{H}$ NMR $\left(\mathrm{CDCl}_{3}, 400 \mathrm{MHz}\right): \delta 1.26(\mathrm{~s}, 12 \mathrm{H}), 1.77-1.84(\mathrm{~m}, 2 \mathrm{H}), 2.14(\mathrm{q}, J=$ $7.2 \mathrm{~Hz}, 4 \mathrm{H}), 5.66$ (s, 1H), $5.86(\mathrm{~d}, J=2.8 \mathrm{~Hz}, 1 \mathrm{H},) ;{ }^{13} \mathbf{C}$ NMR (CDCl3): $\delta 16.4,24.7,24.98,34.3$, 
83.5, 131.1; MS (m/z) (rel. intensity): 220 (9), 206 (33), 135 (100). The physical data were identical to those previously reported data.

\section{4,4,5,5-tetramethyl-2-(1-(p-tolyl)vinyl)-1,3,2-dioxaborolane:}

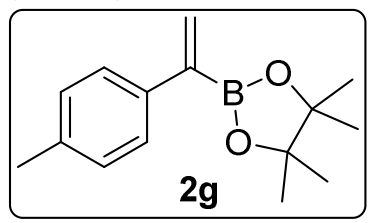

Purified by flash chromatography on silica gel using hexane as eluent. $\mathrm{R}_{f}$ (hexane) $=0.5$. Colourless oil; Yield: 93\%, (280 mg); IR (neat, $\mathrm{cm}^{-1}$ ): 3462, 2959, 1567, 1473, 1451, 1178, 1095, 1018; ${ }^{1} \mathbf{H}$ NMR $\left(\mathrm{CDCl}_{3}, 400 \mathrm{MHz}\right): \delta 7.37$ (d, $\left.J=7.6 \mathrm{~Hz}, 2 \mathrm{H}\right), 7.12$ (d, $\left.J=7.6 \mathrm{~Hz}, 2 \mathrm{H}\right), 6.0$ $(\mathrm{m}, 2 \mathrm{H}), 2.32(\mathrm{~s}, 3 \mathrm{H}), 1.31(\mathrm{~s}, 12 \mathrm{H}) ;{ }^{13} \mathbf{C} \mathbf{N M R}\left(\mathrm{CDCl}_{3}\right): \delta$ 136.6, 129.9, 128.9, 127.0, 124.7, 3.7, 24.7, 21.1; HRMS (ESI) : calculated for $\mathrm{C}_{15} \mathrm{H}_{21} \mathrm{BO}_{2} \mathrm{Na}: 267.1532$; Found: 267.1552.

\section{4,4,5,5-tetramethyl-2-(3-phenoxyprop-1-en-2-yl)-1,3,2-dioxaborolane: ${ }^{5}$}

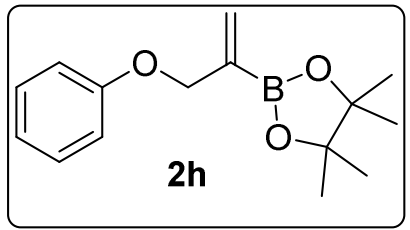

Purified by flash chromatography on silica gel using EtOAc : hexane (1:10) as eluent. $\mathrm{R}_{f}$ (hexane) $=0.5$. Colourless liquid; Yield: $62 \%,(161 \mathrm{mg}) ;$ IR (neat, $\left.\mathrm{cm}^{-1}\right): 3431,2980,2670$, 2336, 2107, 1826, 1347, 1315, 1143; ${ }^{1} \mathbf{H}$ NMR $\left(\mathrm{CDCl}_{3}, 400 \mathrm{MHz}\right): \delta$ 7.28-7.24 (m, 3H), 6.94$6.90(\mathrm{~m}, 2 \mathrm{H}), 6.02(\mathrm{~d}, J=10 \mathrm{~Hz}, 2 \mathrm{H}), 4.6(\mathrm{~s}, 2 \mathrm{H}), 1.28(\mathrm{~s}, 12 \mathrm{H}) ;{ }^{13} \mathbf{C}$ NMR $\left(\mathrm{CDCl}_{3}\right): \delta 24.7,69.1$, 88.3, 83.6, 114.6, 117.2, 120.5, 129.2, 129.4, 129.5, 129.8, 158.7; MS (m/z) (rel. intensity): 260 (51), 245 (53), 175 (100), 133 (15), 93 (29). The physical data were identical to those previously reported data.

2-(3-(((3S,8S,9S,10R,13S,14R,17R)-10,13-dimethyl-17-((R)-6-methylheptan-2-yl)-

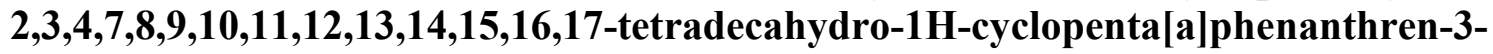
yl)oxy)prop-1-en-2-yl)-4,4,5,5-tetramethyl-1,3,2-dioxaborolane:

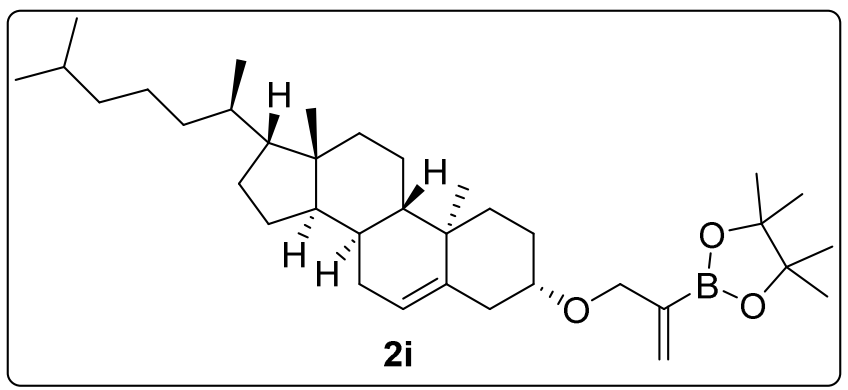

Purified by flash chromatography on silica gel using EtOAc : hexane (1:10) as eluent. $\mathrm{R}_{f}$ (hexane) $=0.5$. Colourless liquid; Yield: 41\%, (226 mg); IR (neat, $\left.\mathrm{cm}^{-1}\right): 3402,2939,1567$, 1484, 1400, 1168, 1065, 1038; ${ }^{1} \mathbf{H}$ NMR $\left(\mathrm{CDCl}_{3}, 400 \mathrm{MHz}\right): 0.67$ (s, 8H), 0.5-0.87 (m, 8H), 0.9$1.00(\mathrm{~m}, 6 \mathrm{H}), 1.10-1.11(\mathrm{~m}, 5 \mathrm{H}), 1.26(\mathrm{~s}, 12 \mathrm{H}), 1.34-1.48(\mathrm{~m}, 12 \mathrm{H}), 1.82-1.99(\mathrm{~m}, 6 \mathrm{H}), 3.2(\mathrm{~m}$, 
1H), $4.13(\mathrm{~s}, 2 \mathrm{H}), 5.33(\mathrm{~s}, 1 \mathrm{H}), 5.91(\mathrm{~m}, 2 \mathrm{H}) ;{ }^{13} \mathbf{C} \mathbf{N M R}\left(\mathrm{CDCl}_{3}\right): \delta$ 11.8, 18.7, 19.3, 21.0, 22.5, 23.8, 24.27, 24.84, 27.99, 28.2, 29.69, 31.9, 35.7, 36.1, 36.8, 39.7, 42.2, 50.15, 56.1, 56.7, 69.4, 83.17, 128.2, 129.0, 130.0, 131.5; MS (m/z) (rel. intensity): 551, 441 (100). (Our attempts to record HRMS for this sample was not successful).

2-(4,4,5,5-tetramethyl-1,3,2-dioxaborolan-2-yl)allyl 2-phenylacetate:

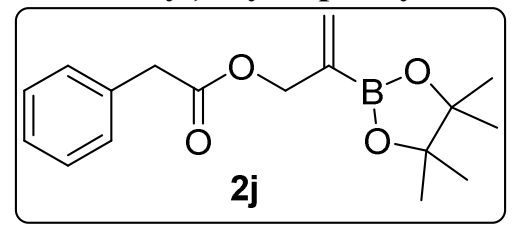

Purified by flash chromatography on silica gel using EtOAc: hexane (1:10) as eluent. $\mathrm{R}_{f}$ (hexane) $=0.5$. Colourless liquid; Yield: $93 \%,\left(280 \mathrm{mg}\right.$ ); IR (neat, $\left.\mathrm{cm}^{-1}\right): 3462,2959,1567,1473,1451$, $1178,1095,1018 ;{ }^{1} \mathbf{H}$ NMR $\left(\mathrm{CDCl}_{3}, 400 \mathrm{MHz}\right): \delta 7.35-7.14(\mathrm{~m}, 11 \mathrm{H}), 6.6(\mathrm{dt}, J=18 \mathrm{~Hz}, J=4.8$ $\mathrm{Hz}, 1.3 \mathrm{H}), 5.9(\mathrm{~s}, 1 \mathrm{H}), 5.75(\mathrm{~s}, 1 \mathrm{H}), 5.65(\mathrm{~d}, J=18 \mathrm{~Hz}, 1.3 \mathrm{H}), 4.72(\mathrm{~s}, 2 \mathrm{H}), 4.67$ (d, J3.6 Hz, 2.8H), $3.66(\mathrm{~s}, 5 \mathrm{H}), 1.26(\mathrm{~s}, 15 \mathrm{H}), 1.24(\mathrm{~s}, 12 \mathrm{H}) ;{ }^{13} \mathbf{C}$ NMR (CDCl3): $\delta$ 24.6, 24.7, 41.4, 41.4, 65.8, 66.3, 83.4, 83.67, 126.9, 127.4, 128.4, 128.5, 129.2, 130.0, 130.1, 133.7, 134.0, 145.7, 171.0; HRMS (ESI) : calculated for $\mathrm{C}_{17} \mathrm{H}_{23} \mathrm{BO}_{4} \mathrm{Na}: 325.1668$; Found: 325.1672.

4-(4,4,5,5-tetramethyl-1,3,2-dioxaborolan-2-yl)pent-4-en-1-ol: ${ }^{7}$

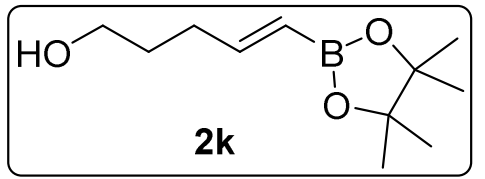

Purified by flash chromatography on silica gel using EtOAc : hexane (1:10) as eluent. $\mathrm{R}_{f}$ (hexane) $=0.5$. Colourless liquid; Yield: 63\%, (133 mg); IR (neat, $\left.\mathrm{cm}^{-1}\right): 3409,2335,2106$, 1887; ${ }^{1} \mathbf{H}$ NMR $\left(\mathrm{CDCl}_{3}, 400 \mathrm{MHz}\right): \delta 6.64\left(\mathrm{dt}, \mathrm{J}_{1}=18 \mathrm{~Hz}, \mathrm{~J}_{2}=6.8,1 \mathrm{H}\right), 6.3-6.29(\mathrm{~m}, 1 \mathrm{H}), 5.47$ $(\mathrm{d}, J=18 \mathrm{~Hz}, 1 \mathrm{H}), 3.71(\mathrm{t}, J=6.8 \mathrm{~Hz}, 2 \mathrm{H}), 3.66(\mathrm{t}, J=6.8 \mathrm{~Hz}, 2 \mathrm{H}), 2.43(\mathrm{q}, J=6.4 \mathrm{~Hz}, 2 \mathrm{H}), 2.25$ (q, $J=7.2 \mathrm{~Hz}, 2 \mathrm{H}), 1.72-1.69(\mathrm{~m}, 6 \mathrm{H}), 1.26$ (s, 24H); ${ }^{13}$ C NMR (CDCl3): $\delta 14.09,24.7,24.79$, 29.6, 13.1, 31.9, 32.2, 61.8, 62.4, 83.2, 141.2, 153.5; MS $(\mathrm{m} / \mathrm{z})$ (rel. intensity): MS (m/z) (rel. intensity): 212 (5), 197 (23), 152 (21), 69 (90), 101 (70), 41(100). The physical data were identical to those previously reported data.

\section{(Z)-3-(4,4,5,5-tetramethyl-1,3,2-dioxaborolan-2-yl)but-2-en-1-ol: ${ }^{8}$}

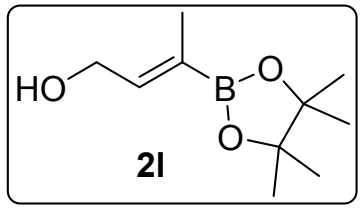

Purified by flash chromatography on silica gel using EtOAc : hexane (1:10) as eluent. $\mathrm{R}_{f}$ (hexane) $=0.5$. Colourless liquid; Yield: 71\%, (140 mg); IR (neat, $\left.\mathrm{cm}^{-1}\right): 3422,2969,1597$, 1454, 1168, 1065; ${ }^{1} \mathbf{H}$ NMR $\left(\mathrm{CDCl}_{3}, 400 \mathrm{MHz}\right): \delta 6.38(\mathrm{~m}, 1 \mathrm{H}), 4.51(\mathrm{~d}, J=5.6 \mathrm{~Hz}, 2 \mathrm{H}), 1.68(\mathrm{~s}$, $3 \mathrm{H}), 1.27(\mathrm{~s}, 12 \mathrm{H}) ;{ }^{13} \mathrm{C}$ NMR $\left(\mathrm{CDCl}_{3}\right): \delta 142.9,83.0,62.1,24.4,14.1 ; \mathbf{M S}(\mathrm{m} / \mathrm{z}): 310 \& 184$; The physical data were identical to those previously reported data. 
(E)-4,4,5,5-tetramethyl-2-(non-1-en-1-yl)-1,3,2-dioxaborolane:

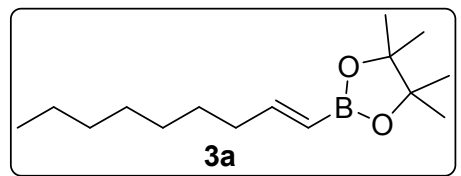

Purified by flash chromatography on silica gel using EtOAc : hexane (1:10) as eluent. $\mathrm{R}_{f}$ (hexane) $=0.5$. yellow liquid; Yield: $80 \%,(201 \mathrm{mg})$; IR (neat, $\left.\mathrm{cm}^{-1}\right): 3392,2979,1591,1478$, 1458, 1188, 1068, 1088; ${ }^{1} \mathbf{H}$ NMR $\left(\mathrm{CDCl}_{3}, 400 \mathrm{MHz}\right): \delta \quad 0.85-0.89(\mathrm{~m}, 3 \mathrm{H}), 1.26(\mathrm{~s}, 12 \mathrm{H}), 1.22-$ $1.34(10 \mathrm{H}, \mathrm{m}), 1.36-1.44(\mathrm{~m}, 2 \mathrm{H}), 2.15(\mathrm{q}, J=8.1 \mathrm{~Hz}, 2 \mathrm{H}), 5.42(\mathrm{~d}, J=18.8 \mathrm{~Hz}, 1 \mathrm{H}), 6.63(\mathrm{dt}, J$ $=18.8,8.1 \mathrm{~Hz}, 1 \mathrm{H},) .{ }^{13} \mathrm{C} \mathbf{N M R}\left(\mathrm{CDCl}_{3}, 125 \mathrm{MHz}\right) \delta=14.1,22.6,24.7,28.2,29.1,29.18,31.79$, 35.8, 82.9, 154.88; MS (m/z) (rel. intensity): 252 (11), 237 (73), 153 (100). (Our attempts to record HRMS for this sample was not successful)

\section{(E)-4,4,5,5-tetramethyl-2-(oct-1-en-1-yl)-1,3,2-dioxaborolane: ${ }^{9}$}

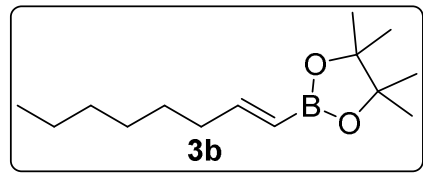

Purified by flash chromatography on silica gel using EtOAc : hexane (1:10) as eluent. $\mathrm{R}_{f}$ (hexane) $=0.5$. pale yellow liquid; Yield: $76 \%,\left(180 \mathrm{mg}\right.$ ); IR (neat, $\left.\mathrm{cm}^{-1}\right): 3467,2928,2617$, 1362, 1318; ${ }^{1} \mathbf{H}$ NMR $\left(\mathrm{CDCl}_{3}, 400 \mathrm{MHz}\right): \delta \quad 0.86-0.89(\mathrm{~m}, 3 \mathrm{H}), 1.26(\mathrm{~m}, 12 \mathrm{H}), 1.22-1.34(\mathrm{~m}$, $8 \mathrm{H}), 1.39-1.41(\mathrm{~m}, 2 \mathrm{H}), 2.15(\mathrm{q}, J=6.4 \mathrm{~Hz}, 2 \mathrm{H}), 5.42(\mathrm{~d}, J=18 \mathrm{~Hz}, 1 \mathrm{H}),$,6.63 (dt, $J=18.8,8.1$ $\mathrm{Hz}, 1 \mathrm{H}) .{ }^{13} \mathrm{C}$ NMR $\left(\mathrm{CDCl}_{3}, 125 \mathrm{MHz}\right) \delta=14.0,22.5,24.7,28.1,28.8,31.6,35.8,82.9,154.8$; MS ( $\mathrm{m} / \mathrm{z})$ (rel. intensity): 238 (10), 223 (53), 153 (100). The physical data were identical to those previously reported data.

(E)-2-(hept-1-en-1-yl)-4,4,5,5-tetramethyl-1,3,2-dioxaborolane: ${ }^{4}$

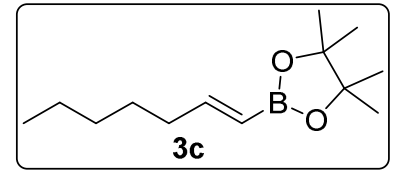

Purified by flash chromatography on silica gel using EtOAc : hexane (1:10) as eluent. $\mathrm{R}_{f}$ (hexane) $=0.5$. yellow liquid; Yield: $85 \%,(190 \mathrm{mg})$; IR (neat, $\left.\mathrm{cm}^{-1}\right): 3658,2974,2927,1638$, 1462; ${ }^{1}$ H NMR $\left(\mathrm{CDCl}_{3}, 400 \mathrm{MHz}\right): \delta$ 0.86-0.89 (m, 3H), $1.26(\mathrm{~s}, 16 \mathrm{H}), 1.39-1.43(\mathrm{~m}, 2 \mathrm{H}), 2.14$ $(\mathrm{q}, J=7.2 \mathrm{~Hz}, 2 \mathrm{H}), 5.42(\mathrm{~d}, J=18 \mathrm{~Hz}, 1 \mathrm{H}), 6.63(\mathrm{dt}, J=18 \mathrm{~Hz}, 1 \mathrm{H}) ;{ }^{13} \mathbf{C} \mathbf{N M R}\left(\mathrm{CDCl}_{3}\right): \delta 13.9$, 22.5, 24.7, 27.8, 31.3, 35.7, 82.9, 154.8; MS (m/z) (rel. intensity): 224 (11), 209 (53), 153 (100). The physical data were identical to those previously reported data.

(E)-4,4,5,5-tetramethyl-2-styryl-1,3,2-dioxaborolane: ${ }^{4}$

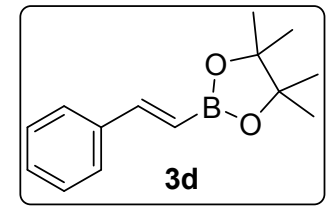

Purified by flash chromatography on silica gel using EtOAc : hexane (1:10) as eluent. $\mathrm{R}_{f}$ (hexane) $=0.5$. Colourless liquid; Yield: 81\%, (186 mg); IR (neat, $\mathrm{cm}^{-1}$ ): 3411, 2973, 1576, 
1479, 1458, 1171, 1043, 1041; ${ }^{1} \mathbf{H}$ NMR $\left(\mathrm{CDCl}_{3}, 400 \mathrm{MHz}\right): \delta 1.31(\mathrm{~s}, 12 \mathrm{H}),, 6.17(1 \mathrm{H}, \mathrm{d}, J=$ $18.8 \mathrm{~Hz}) .{ }^{13} \mathrm{C}$ NMR $(\mathrm{CDCl} 3,125 \mathrm{MHz}) \delta=24.7,83.3,127.0,128.5,128.8,137.4,149.4$; MS $(\mathrm{m} / \mathrm{z})$ (rel. intensity): 230 (51), 215 (53), 146 (100), 103 (91). The physical data were identical to those previously reported data.

\section{References:}

(1) Hintermann, L.; Beilstein J. Org. Chem. 2007, 3: 22; (b) Arduengo, A. J.; Krafczyk, R.; Schmutzler, R.; Craig, H. A.; Goerlich, J. R.; Marshall, W. J.; Unverzagt, M. Tetrahedron 1999, 55, 14523-14534. (c) Bantreil, X.; Nolan, S. P. Nat. Prot. 2011, 6, 69-77.

(2) Yuan, W.; Ma, S. Adv. Syn. Cat. 2012, 354, $1867-1872$.

(3) Ganic, A.; Pfaltz, A. Chem. -A Eur. J. 2012, 18, 6724-6728.

(4) Coombs, J. R.; Zhang, L.; Morken, J. P. Org. Lett. 2015, 17, 1708 - 1711.

(5) Moure, A. L.; Mauleón, P.; Arrayás, R. G.; Carretero, J. C. Org. Lett. 2013, 15, 20542057.

(6) Jun, T.; Kou, T.; Tatsuo, I.; Miyaura, N. J. Am. Chem. Soc. 2002, 124, $8001-8006$.

(7) Jang, H.; Zhugralin, A. R.; Lee, Y.; Hoveyda, A. H. J. Am. Chem. Soc. 2011, 133, 78597871.

(8) Moure, A. L.; Arrayás, R. G.; Cárdenas, D. J.; Alonso, I.; Carretero, J. C. J. Am. Chem. Soc. 2012, 134, 7219-7222.

(9) Takaya, J.; Kirai, N.; Iwasawa, N. J. Am. Chem. Soc. 2011 , 133, 12980 - 12983 
ญั่

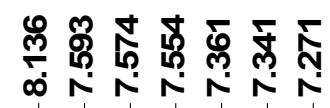

苟
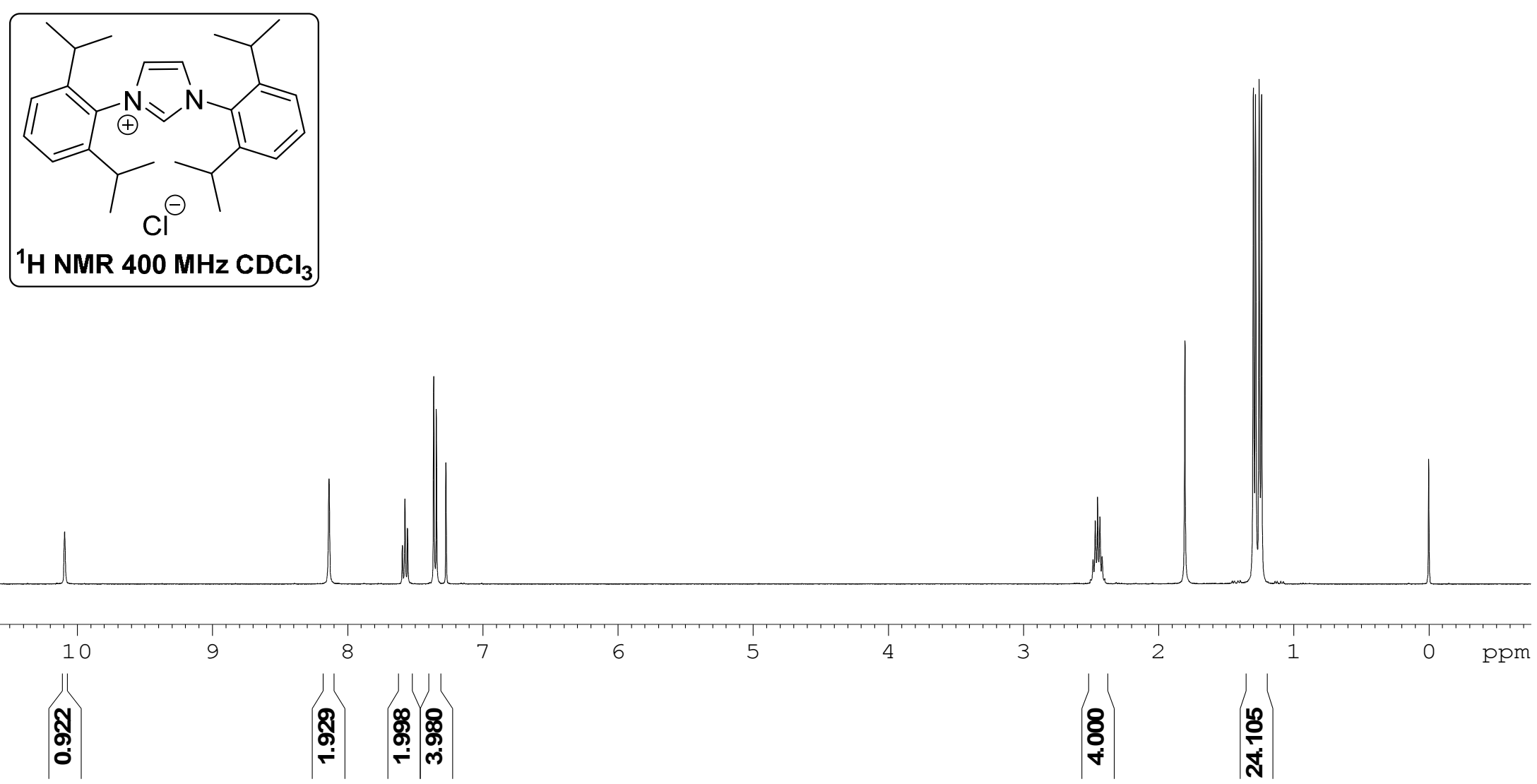


|

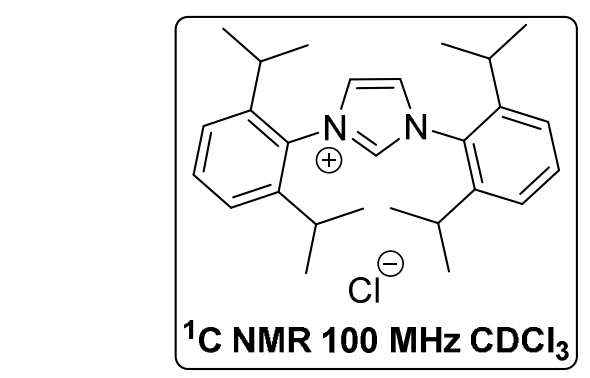



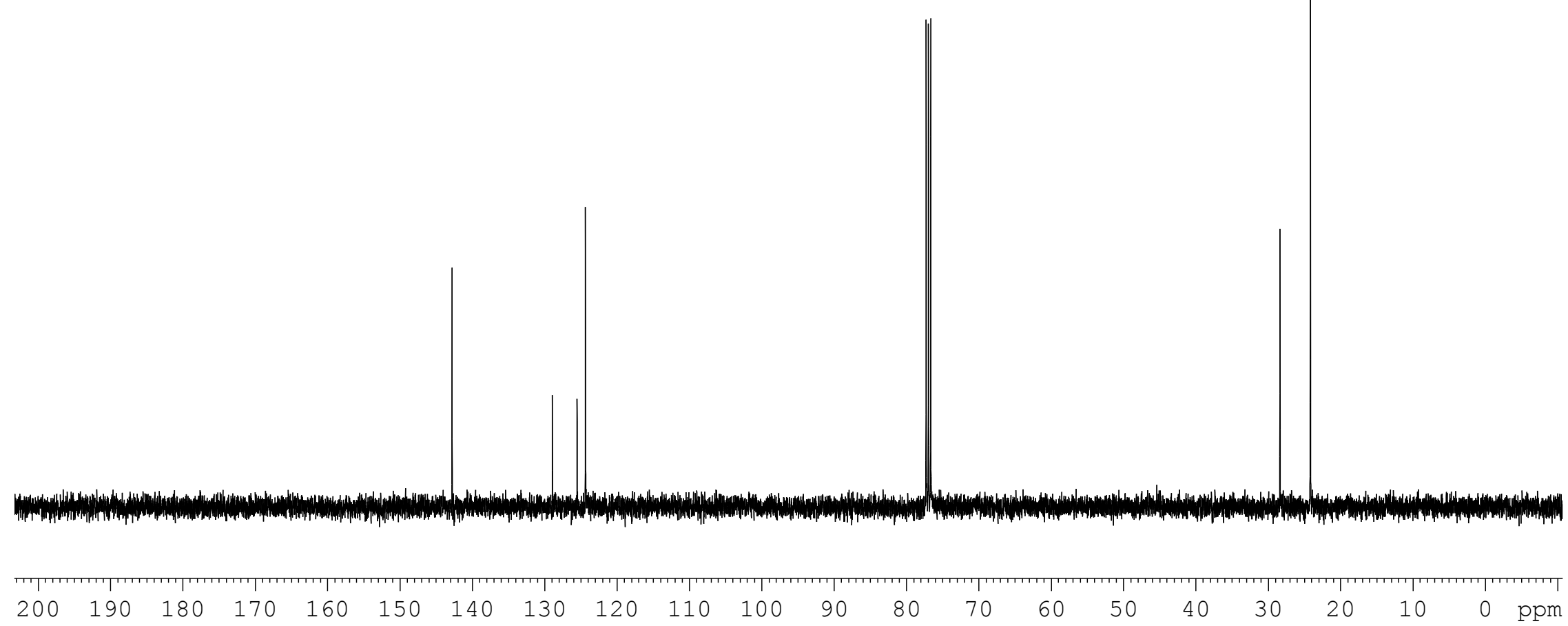




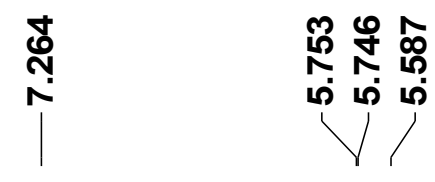

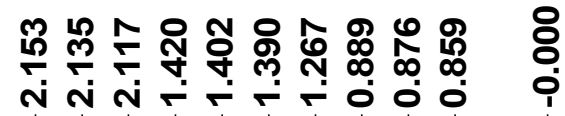
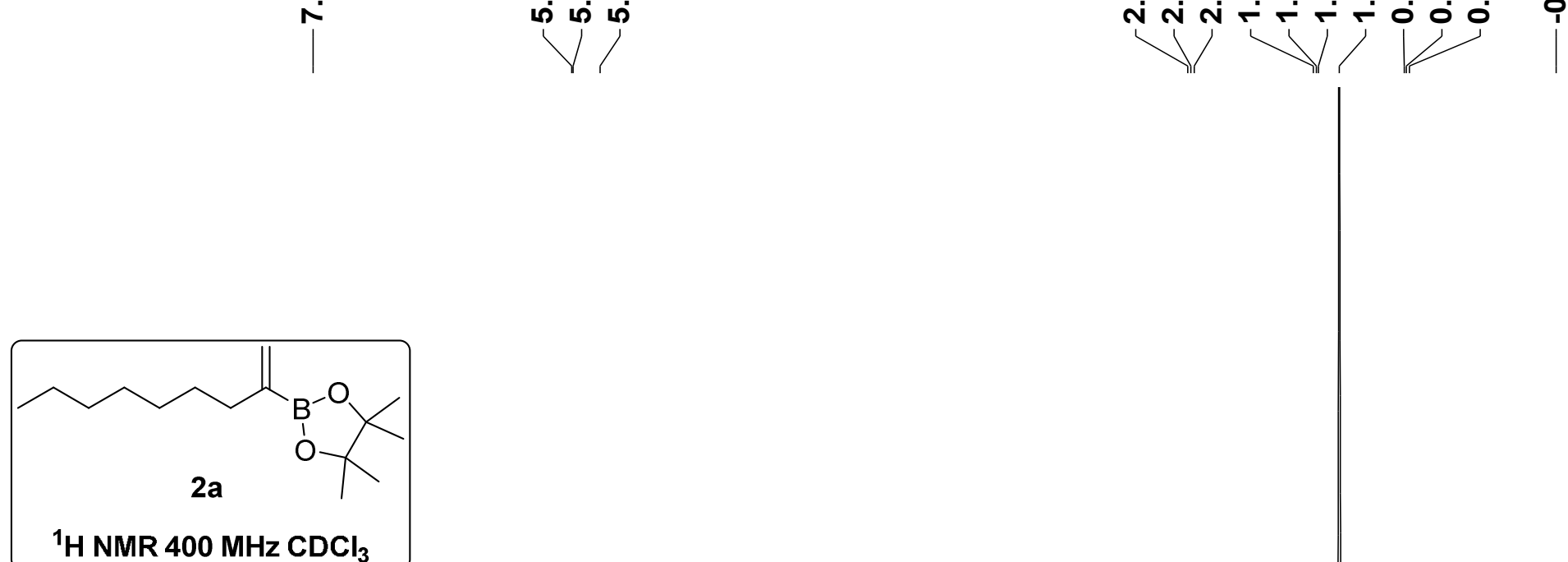

$\mathrm{NMR}_{400} \mathrm{MHz} \mathrm{CDCl}_{3}$

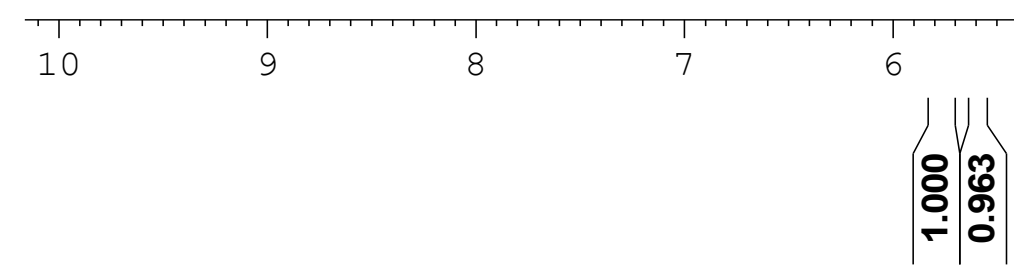




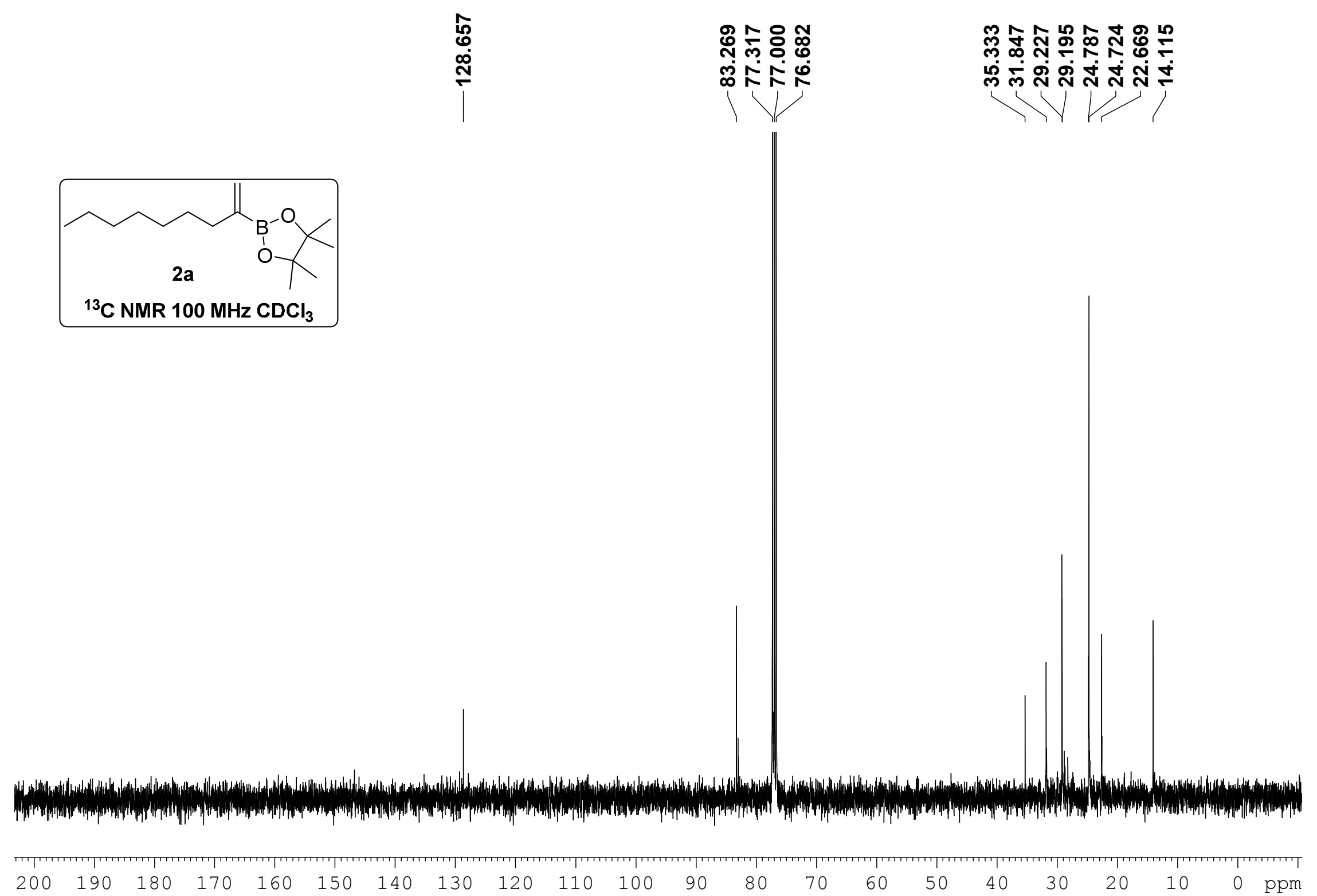




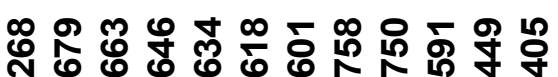

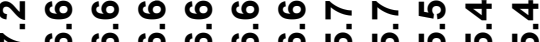

N 000000 in

$\longrightarrow$ VIII

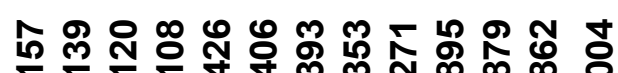

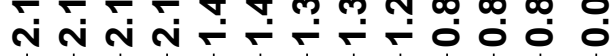

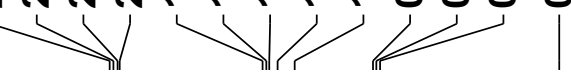

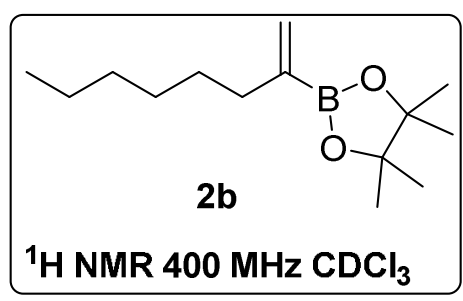

${ }^{1} \mathrm{H} \mathrm{NMR} 400 \mathrm{MHz} \mathrm{CDCl}_{3}$

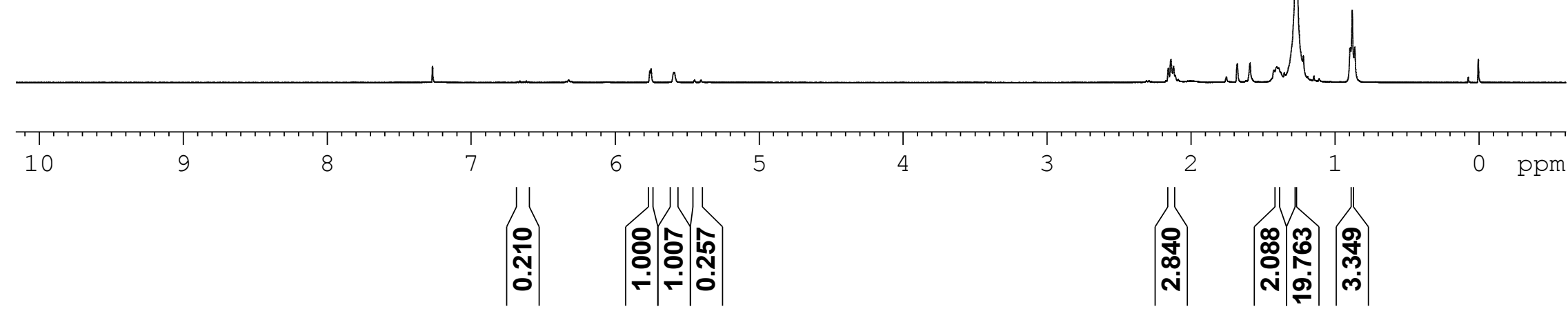




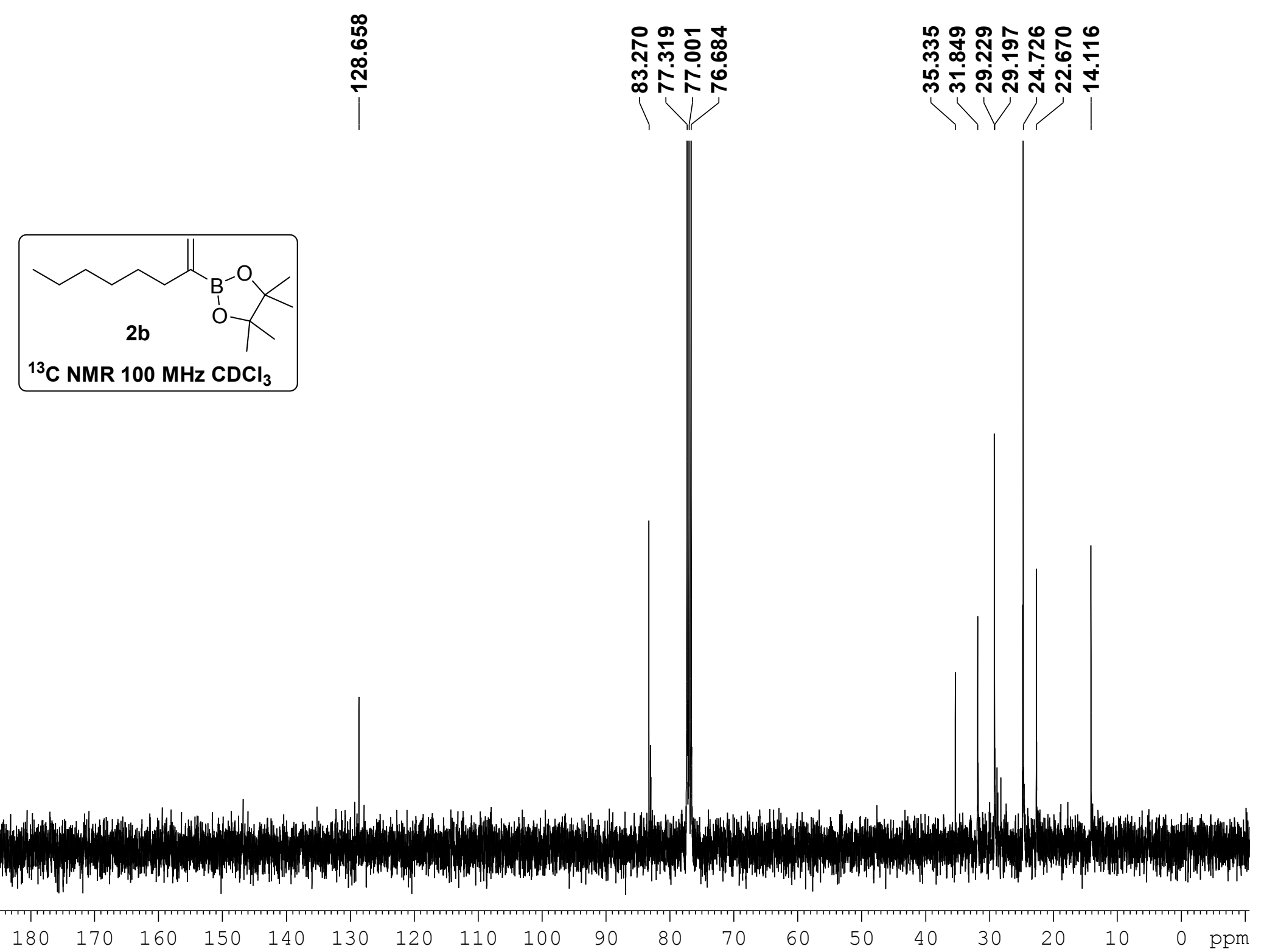




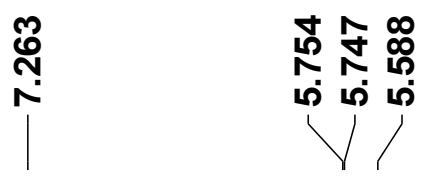

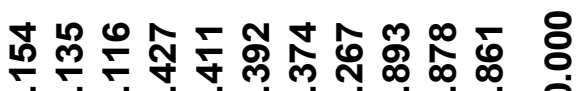

त
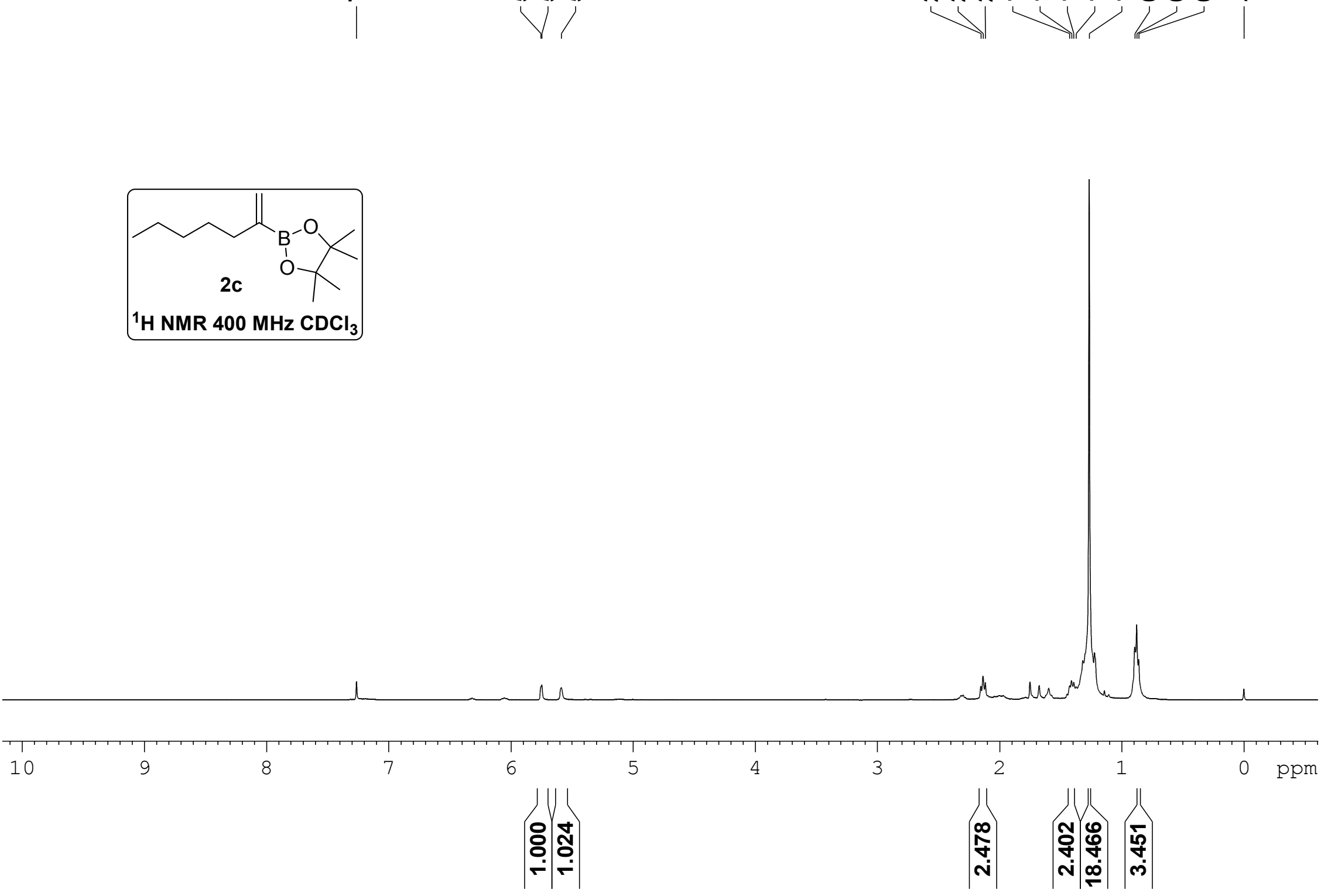


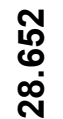

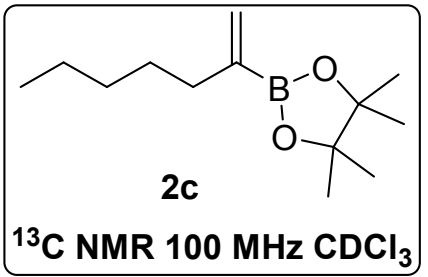

ํำㅇํㅇ :

๓ก゚゚

$1 V$

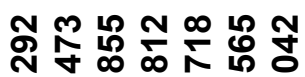

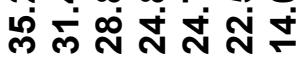

$(n+m)$

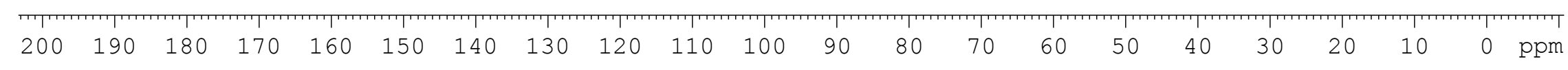

SI - 17 


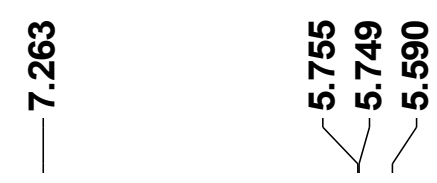

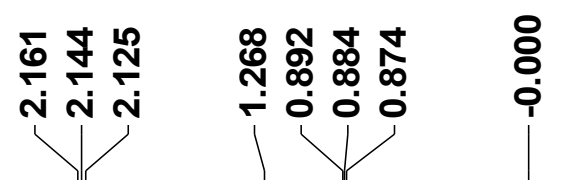
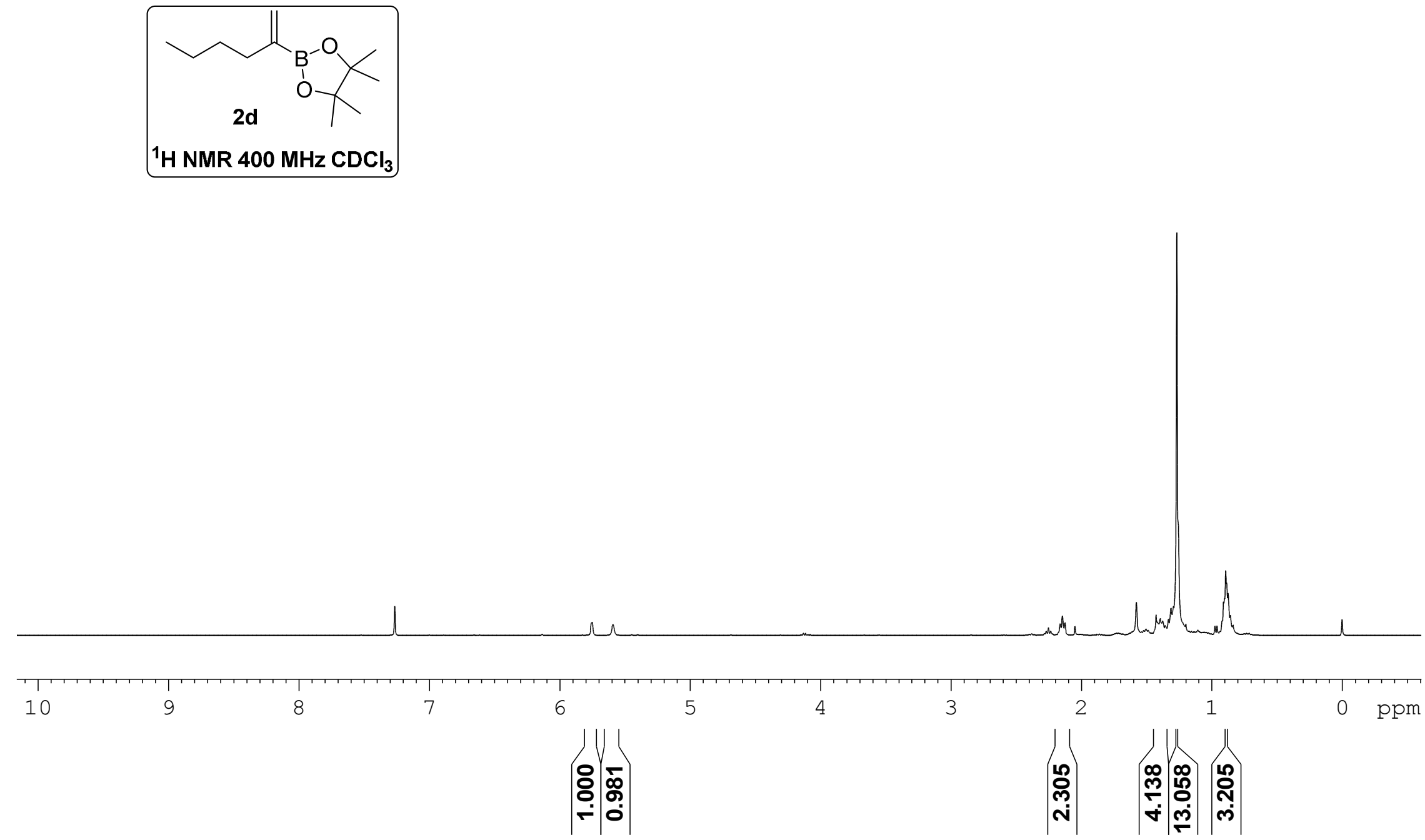


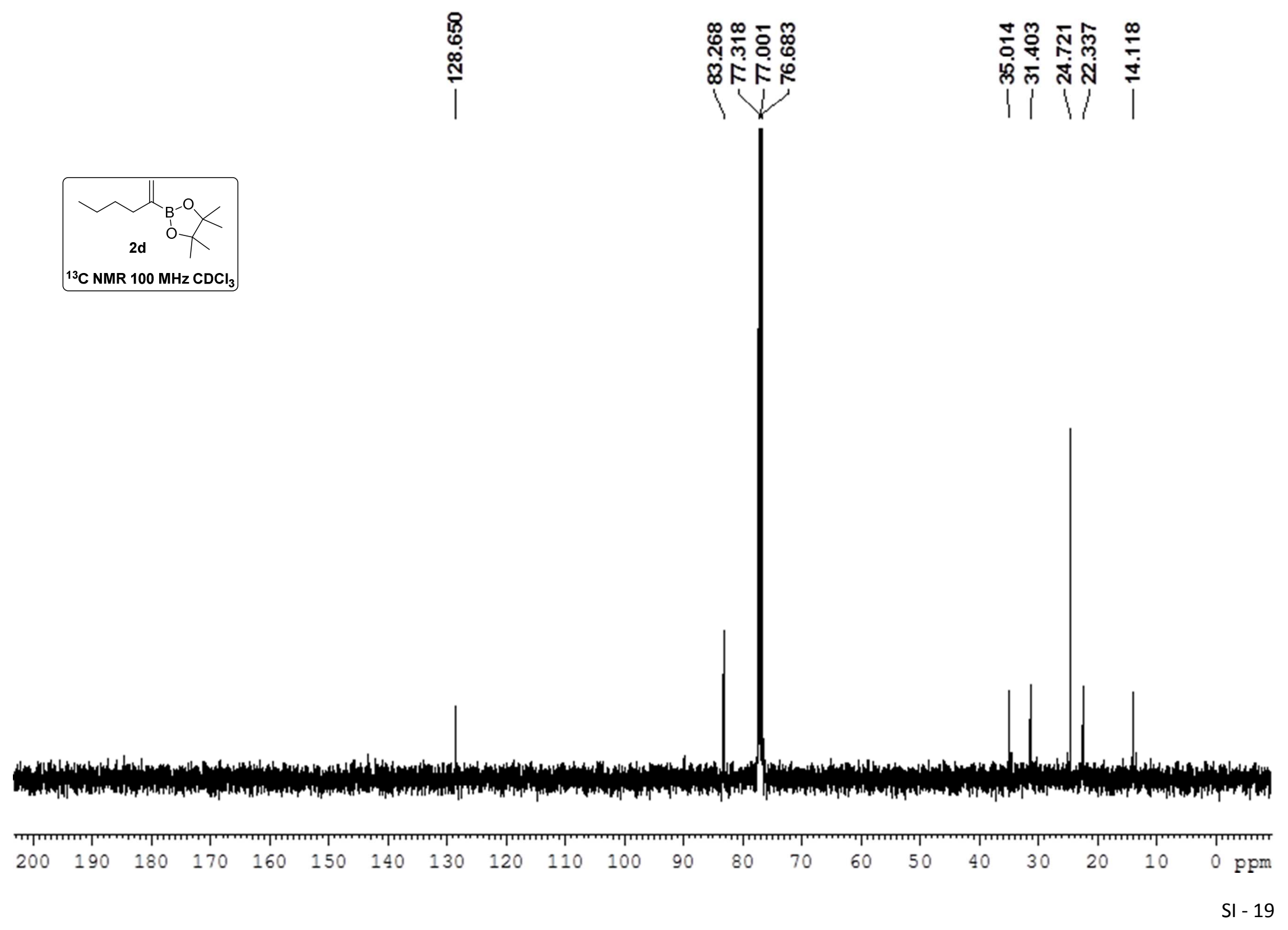


용ำ

ก่ำ

in

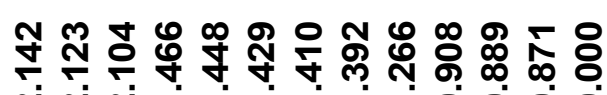

ডั
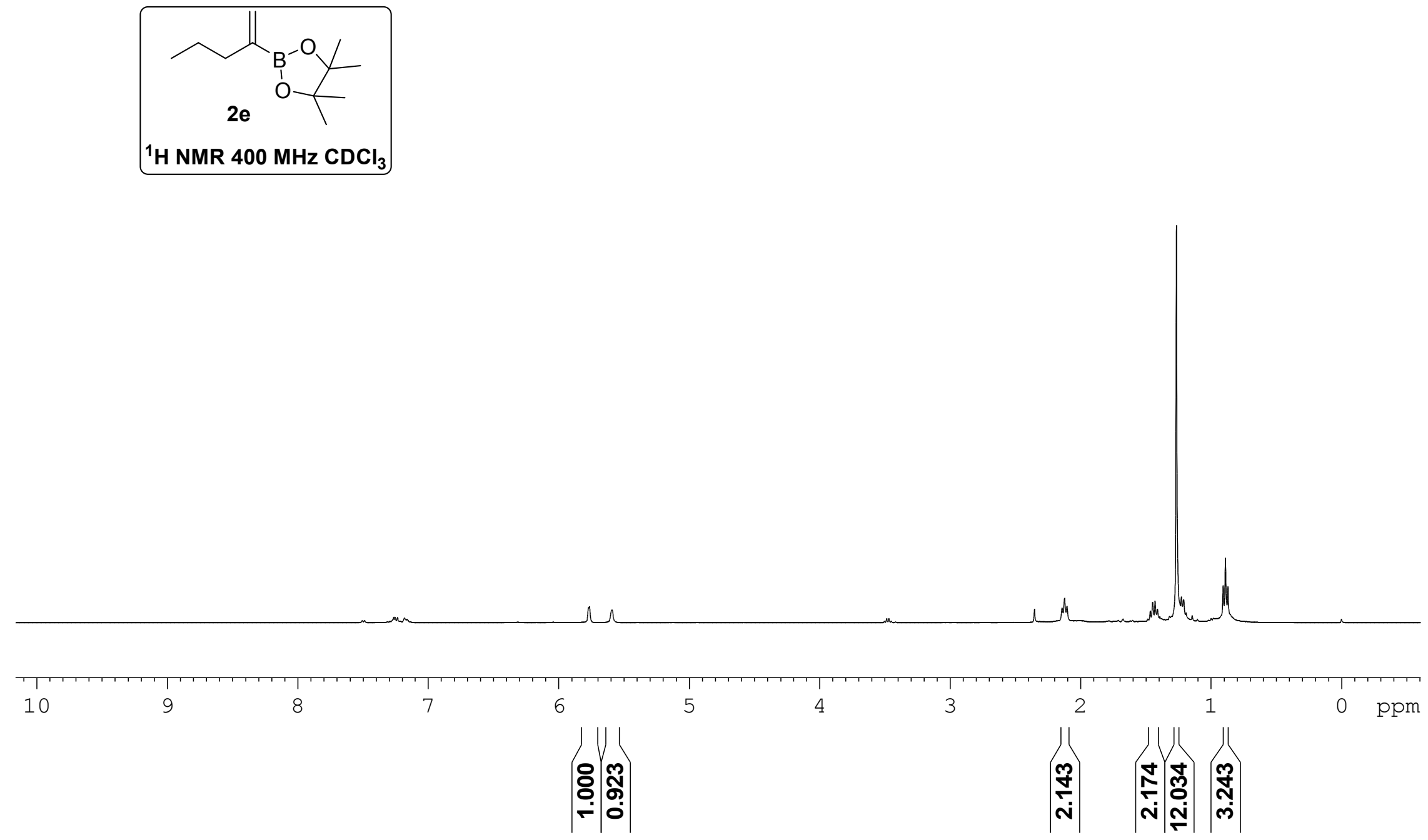
సิำ

令卡

11
ᄂ
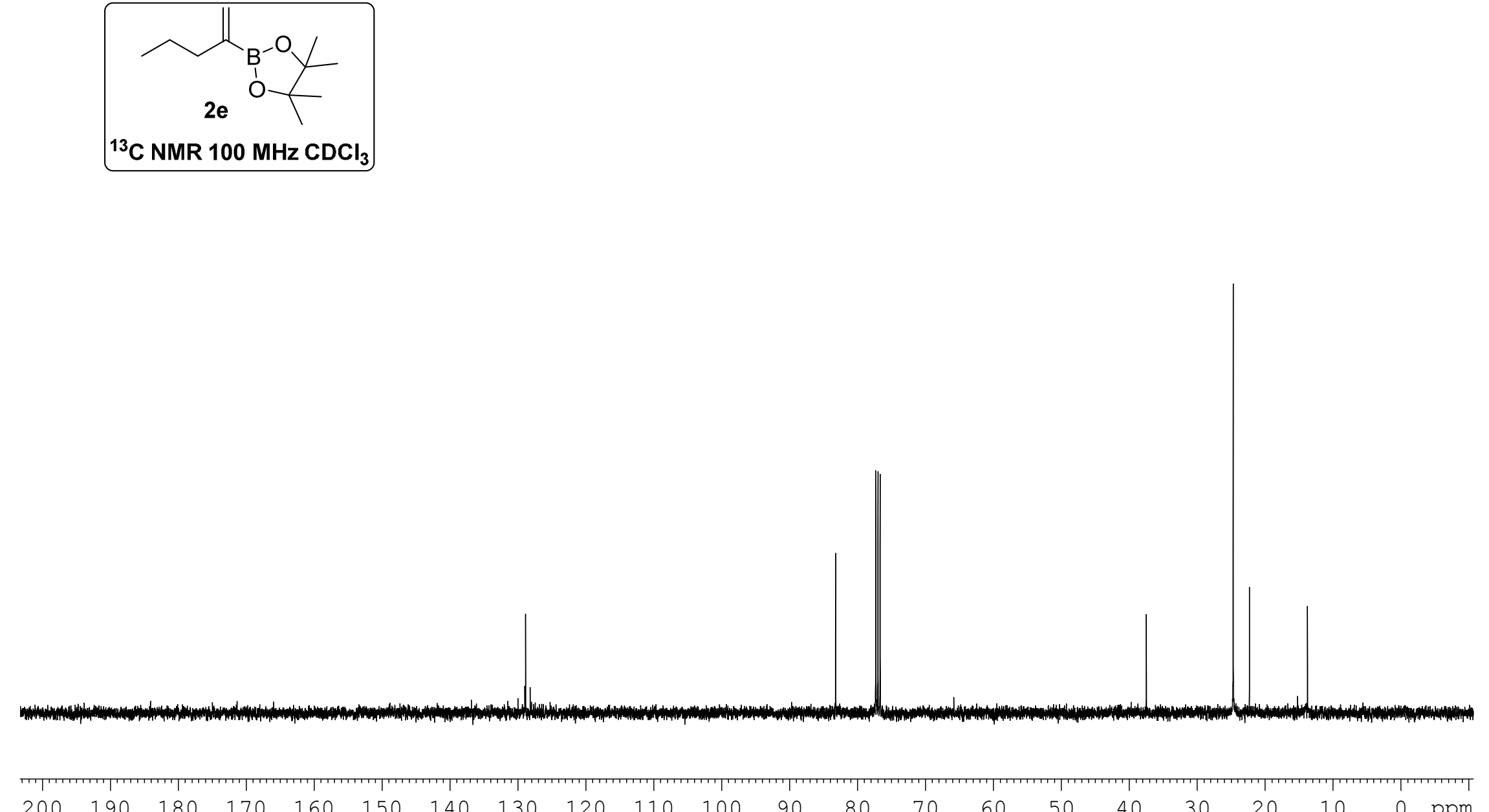


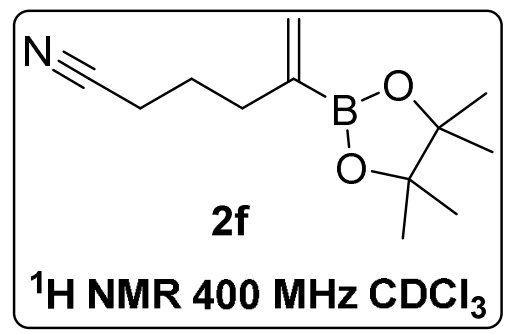



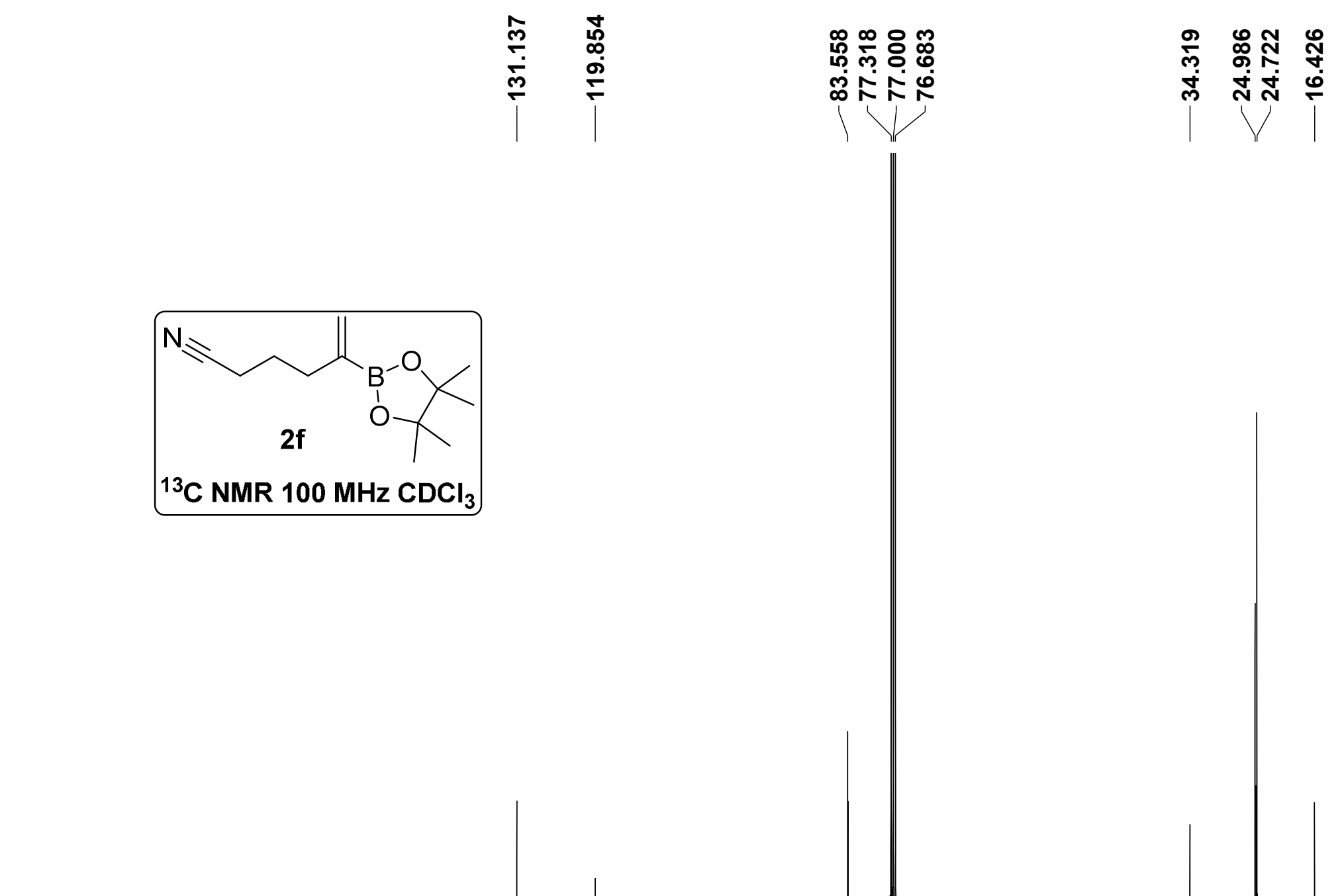


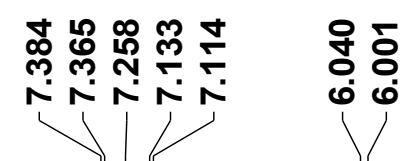

ก)

¿

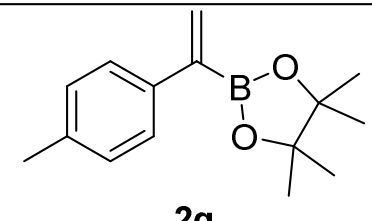

2g

${ }^{1} \mathrm{H}$ NMR $400 \mathrm{MHz} \mathrm{CDCl}_{3}$

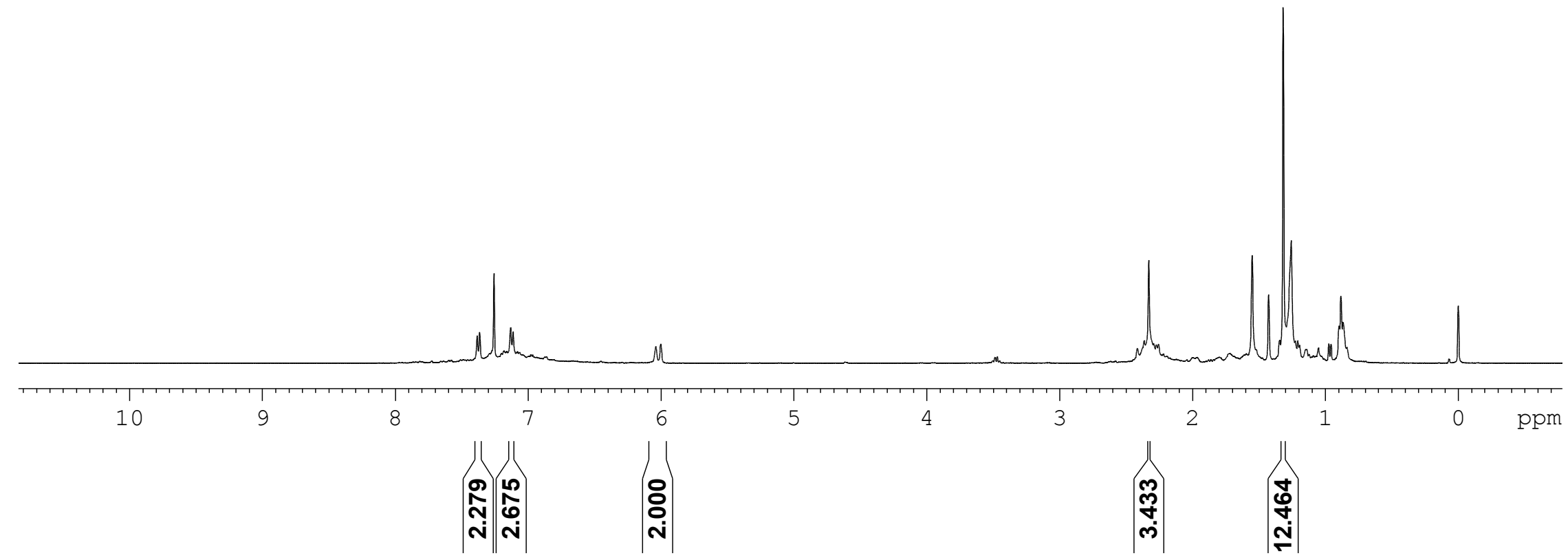



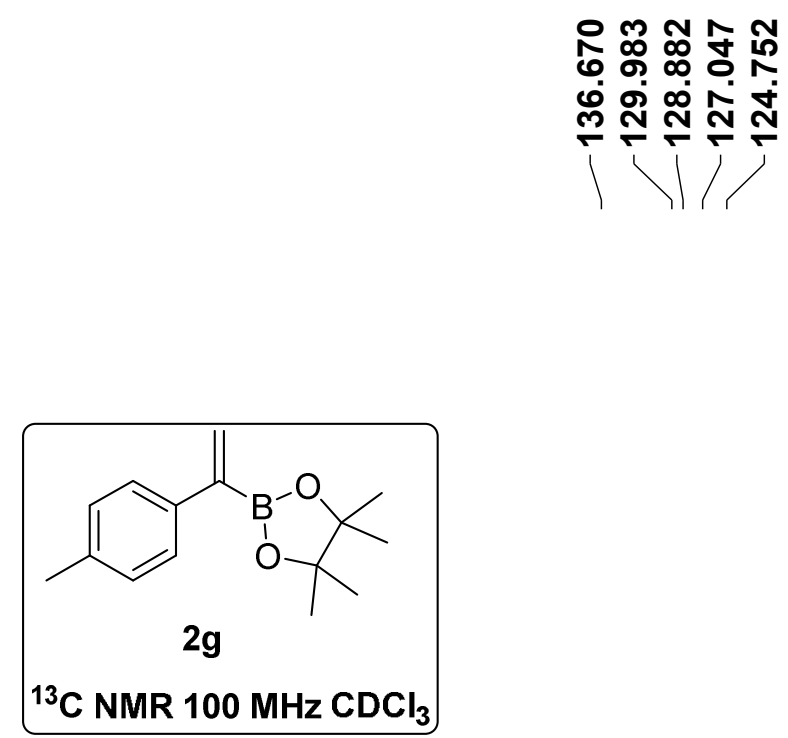

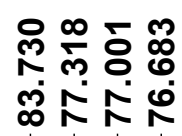

๑5

ํำ

1 V

|

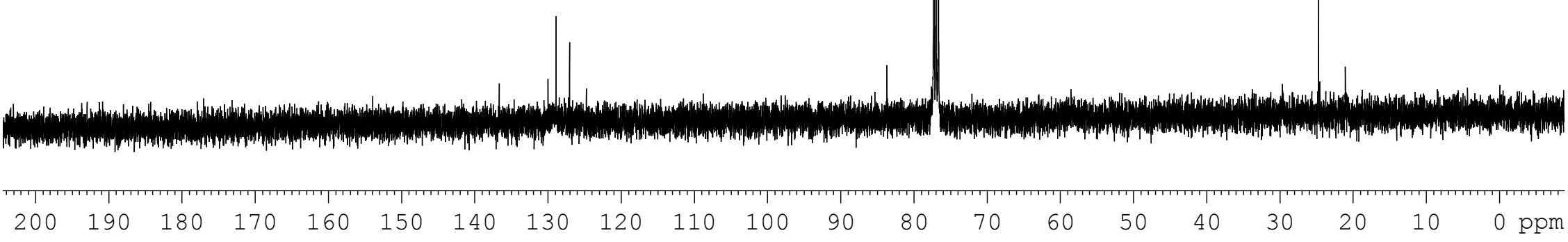


ஜำำ ํํำ

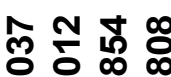
فํ

11

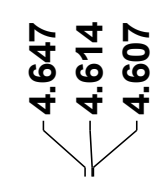

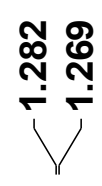

ర̊

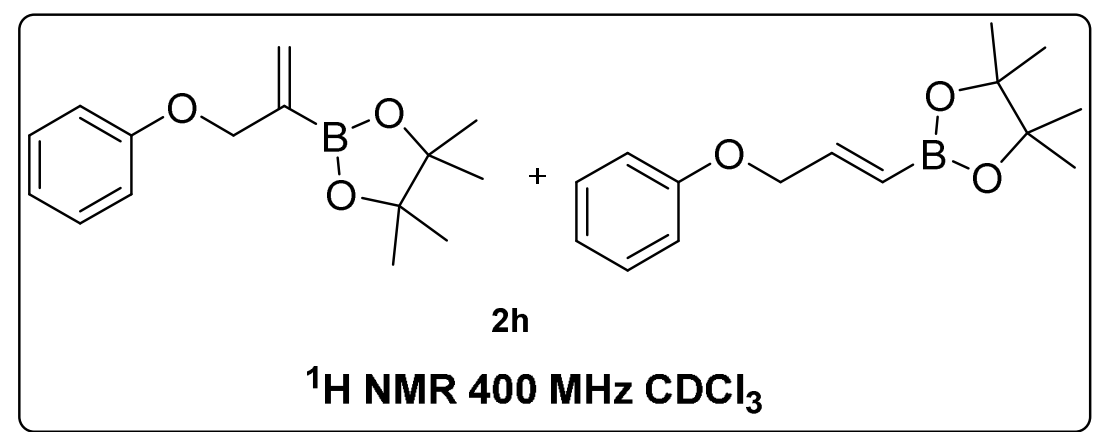

${ }^{1} \mathrm{H}$ NMR $400 \mathrm{MHz} \mathrm{CDCl}_{3}$

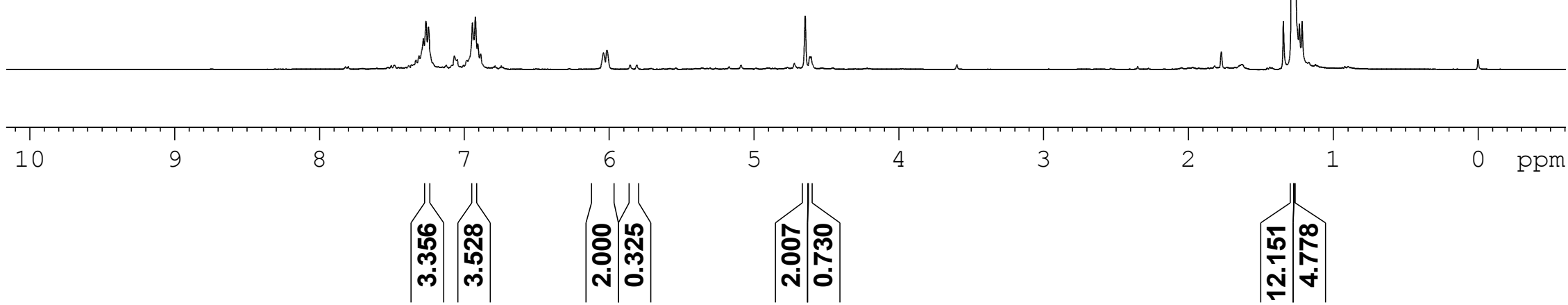




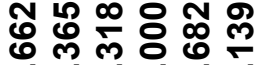

๓

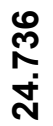

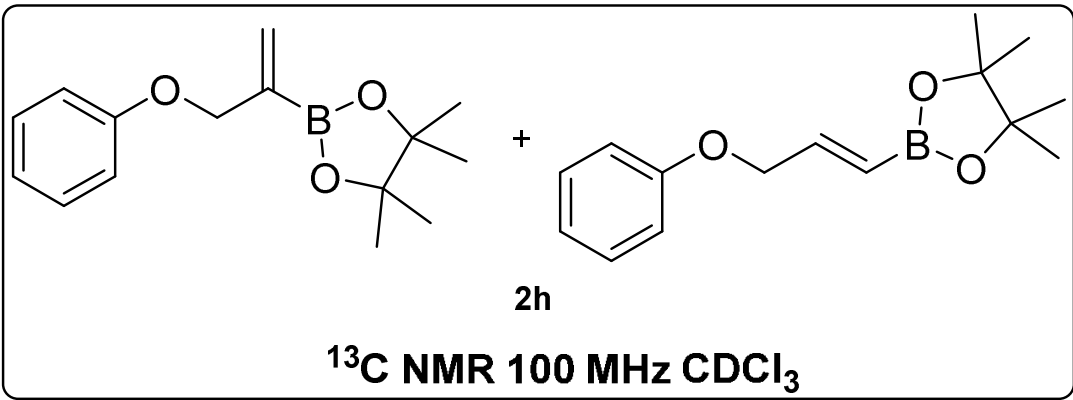

$1 V$

CNMR $100 \mathrm{MHz}_{\mathrm{CDCl}}$

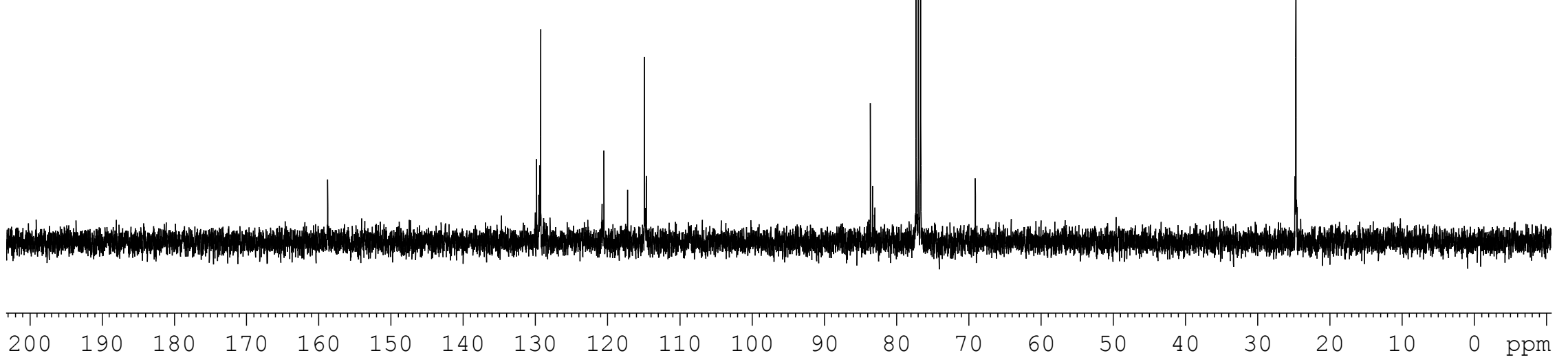




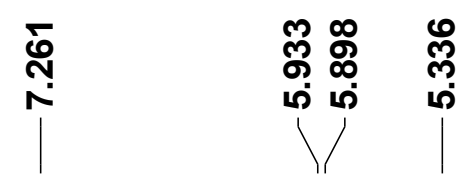

$\stackrel{\bar{m}}{\leftarrow}$

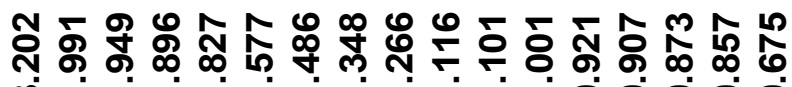

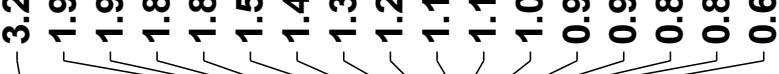
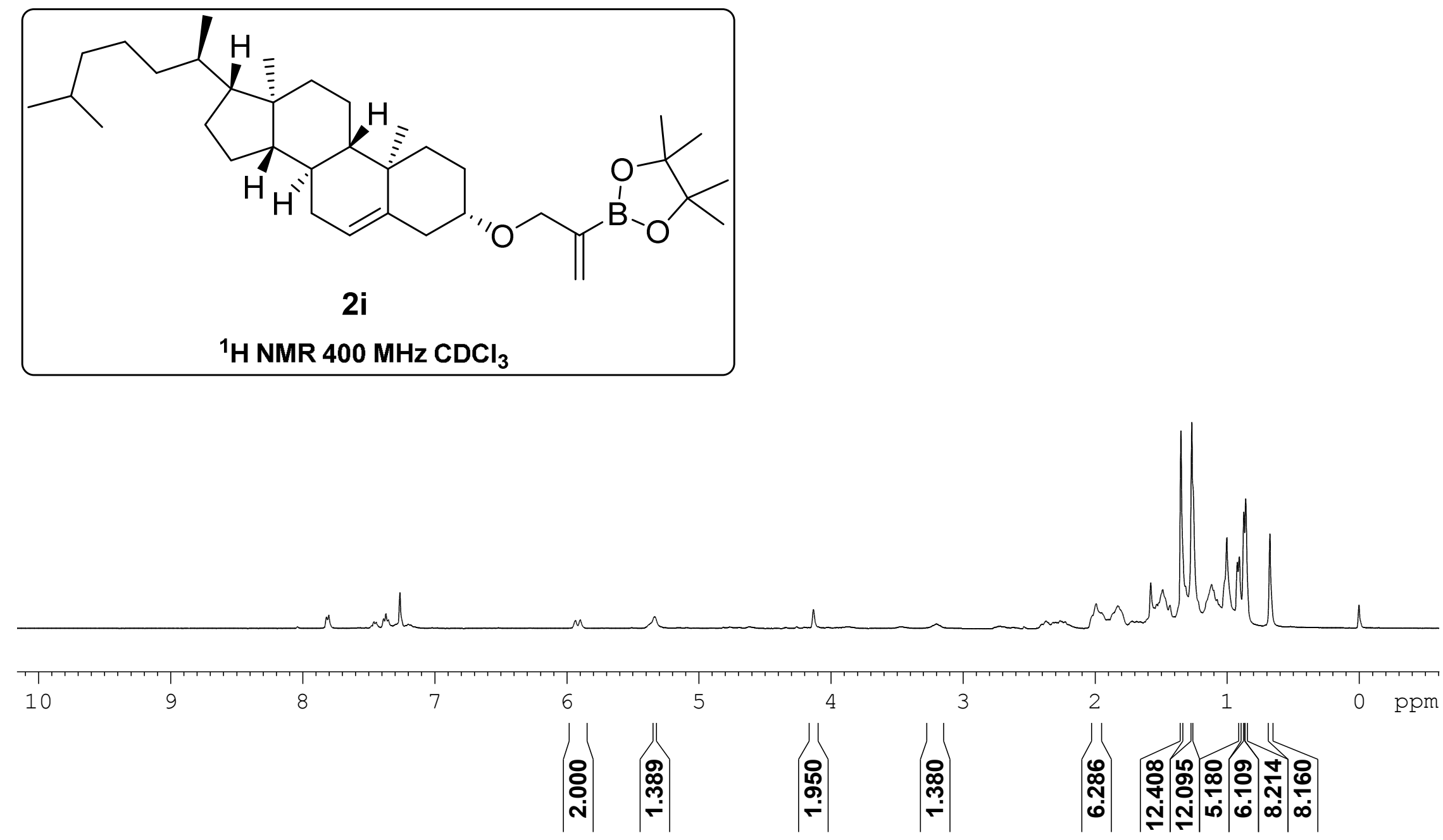


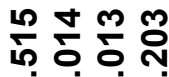

它品 穴

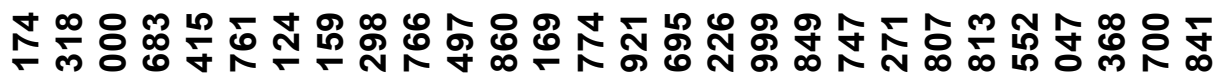

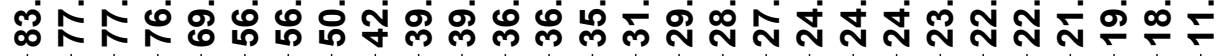

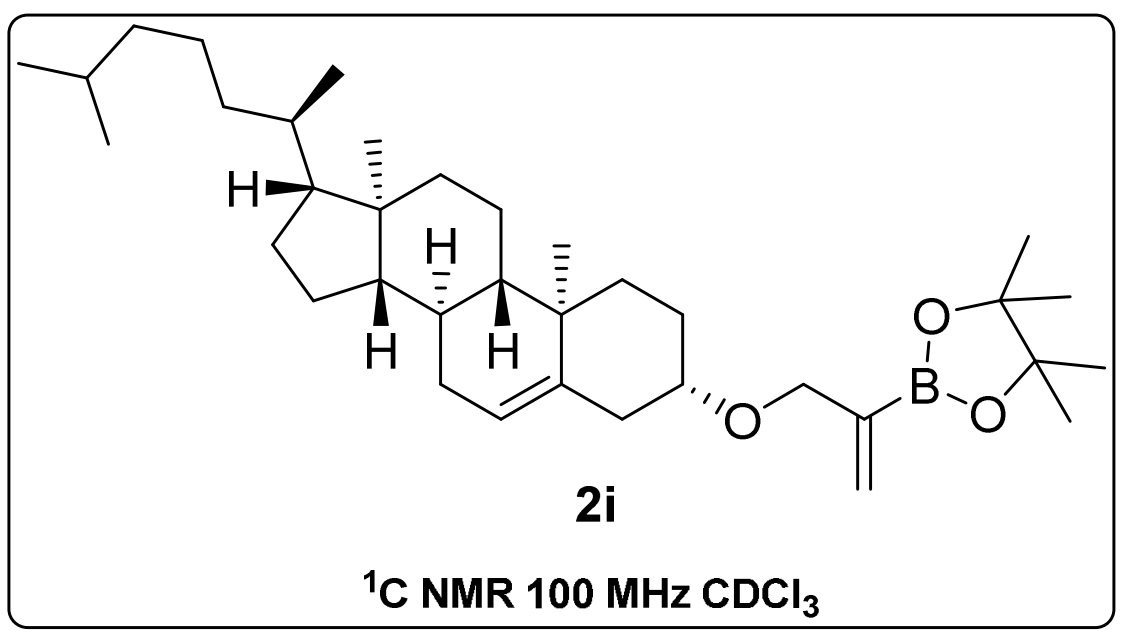

180

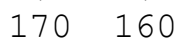

150

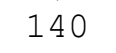

130

120

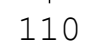

100

90

80

70

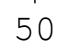

40

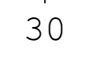

20

10

$-10 \mathrm{ppm}$ 


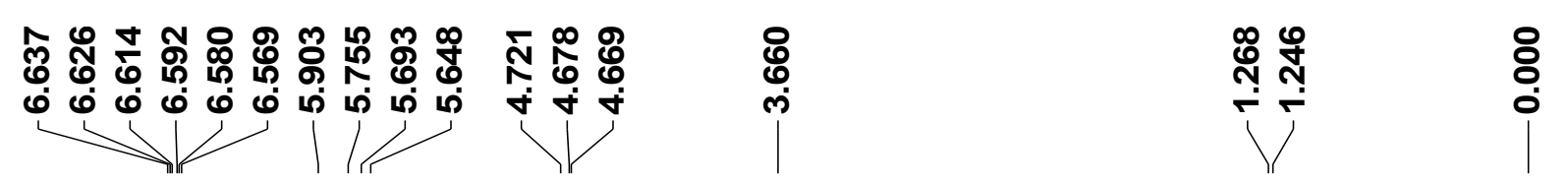
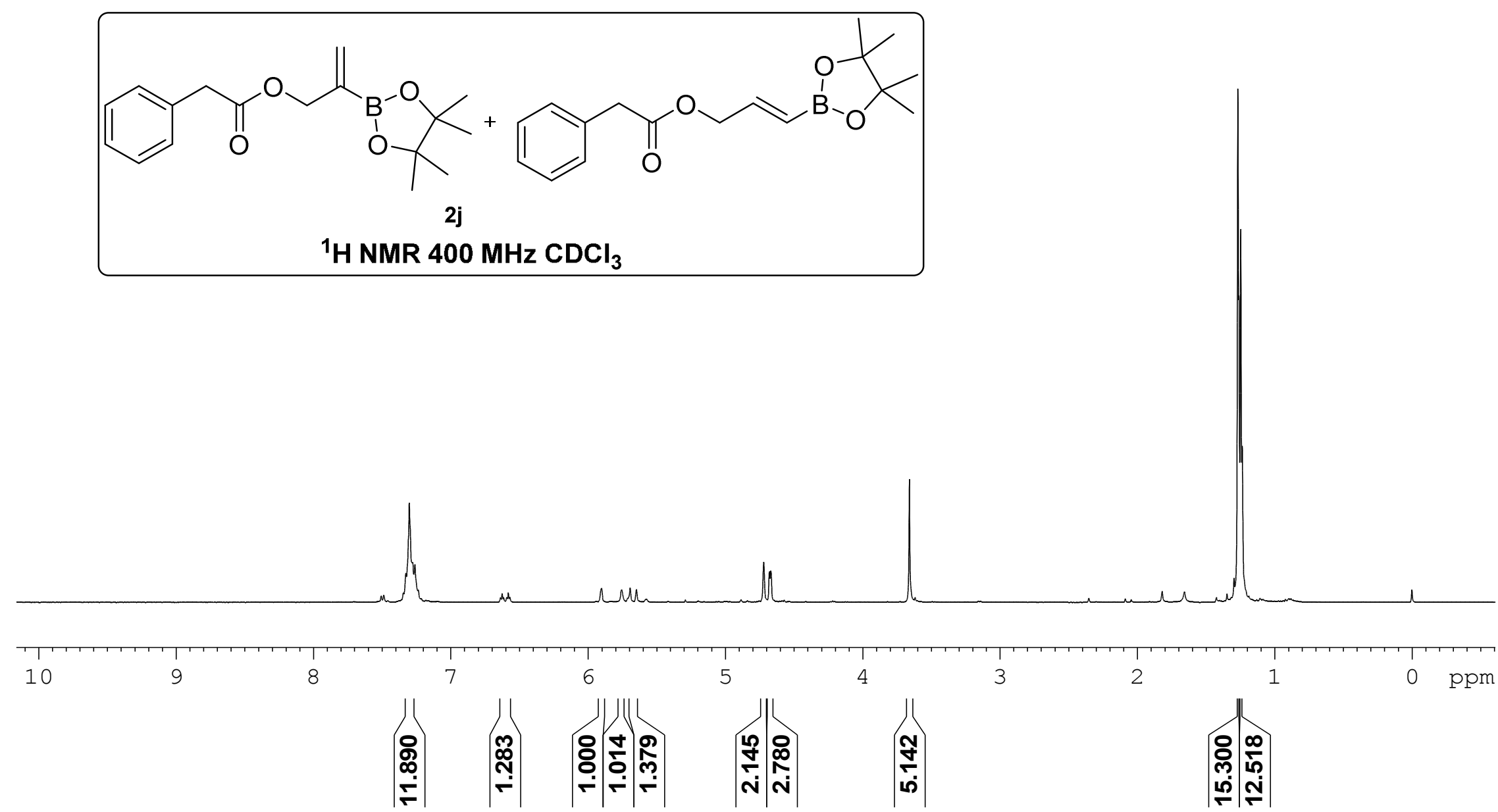


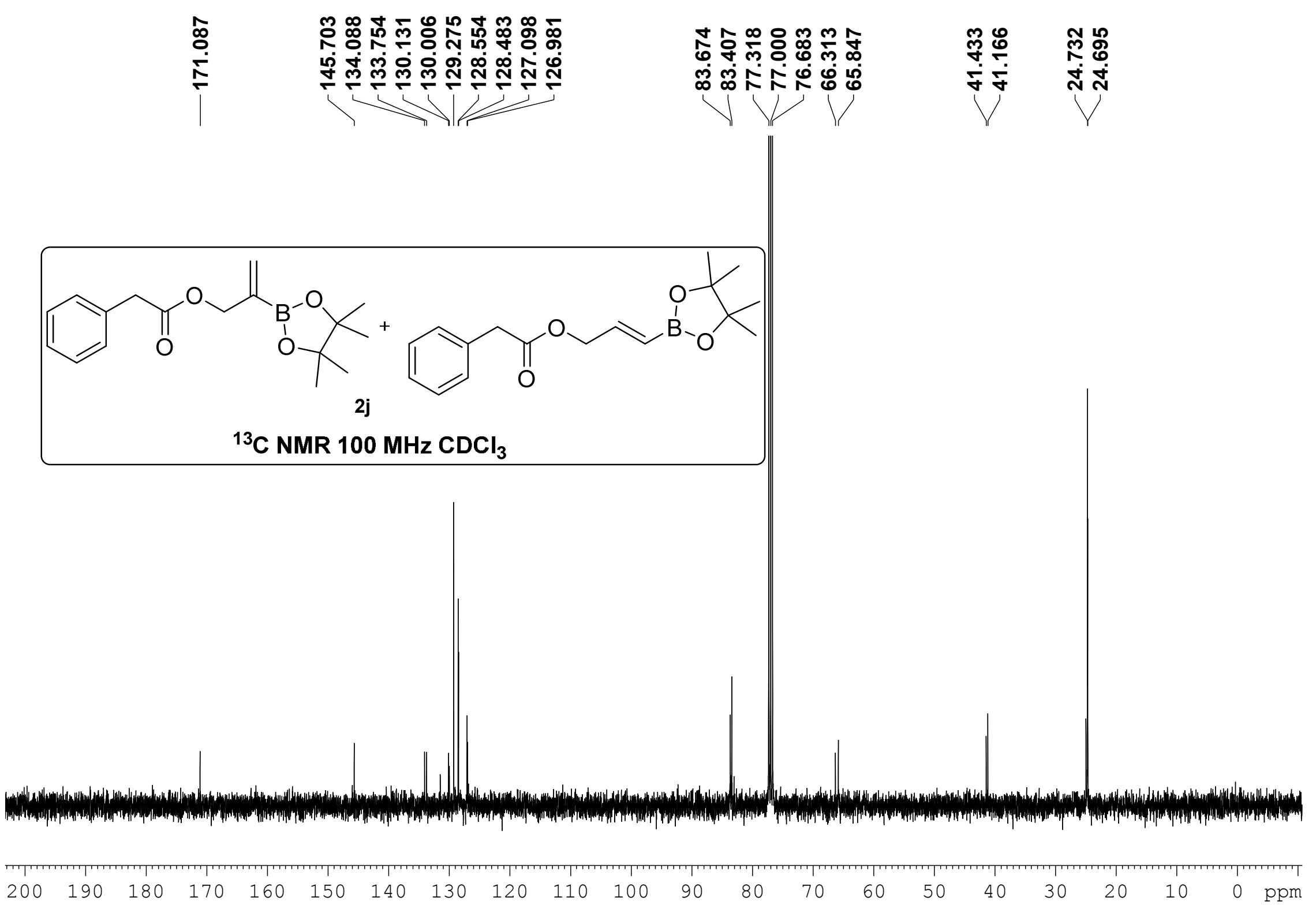




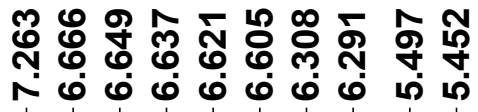

121

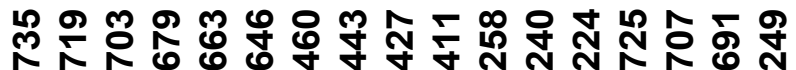
ले

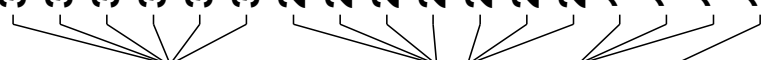

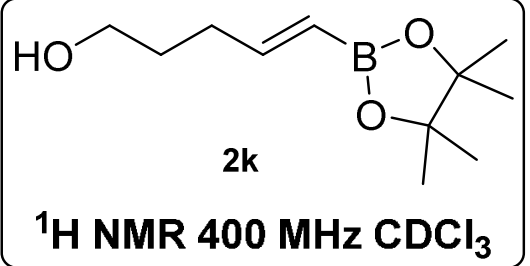

${ }^{1} \mathrm{H}$ NMR $400 \mathrm{MHz} \mathrm{CDCl}_{3}$

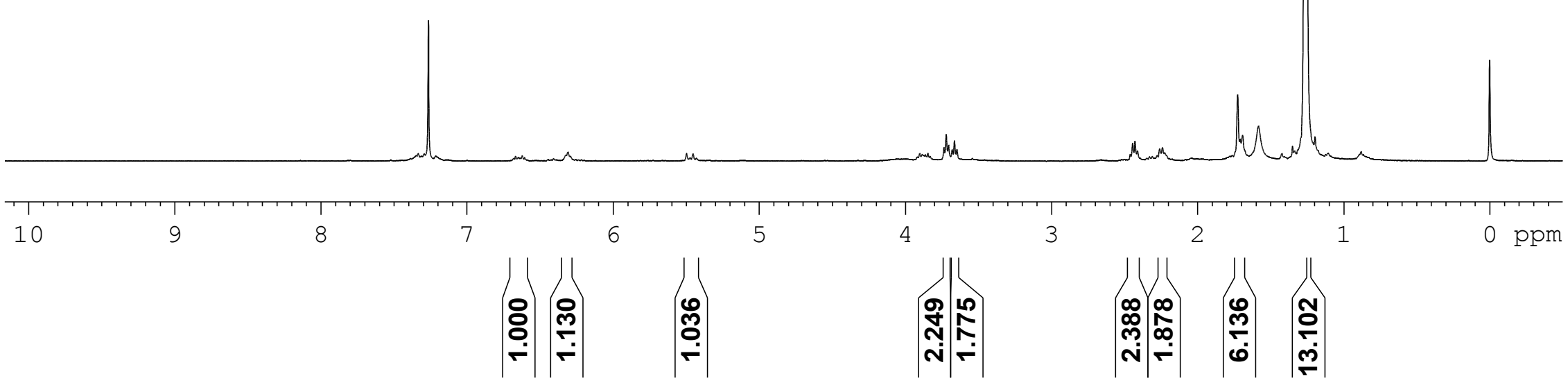




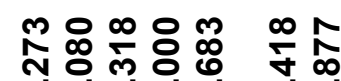

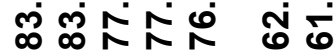

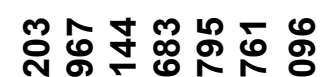

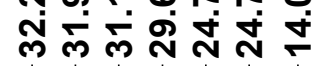

$\backslash / 1$

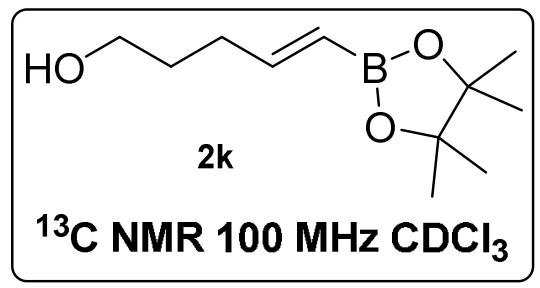

${ }^{13} \mathrm{C}$ NMR $100 \mathrm{MHz}^{\mathrm{CDCl}} 3$
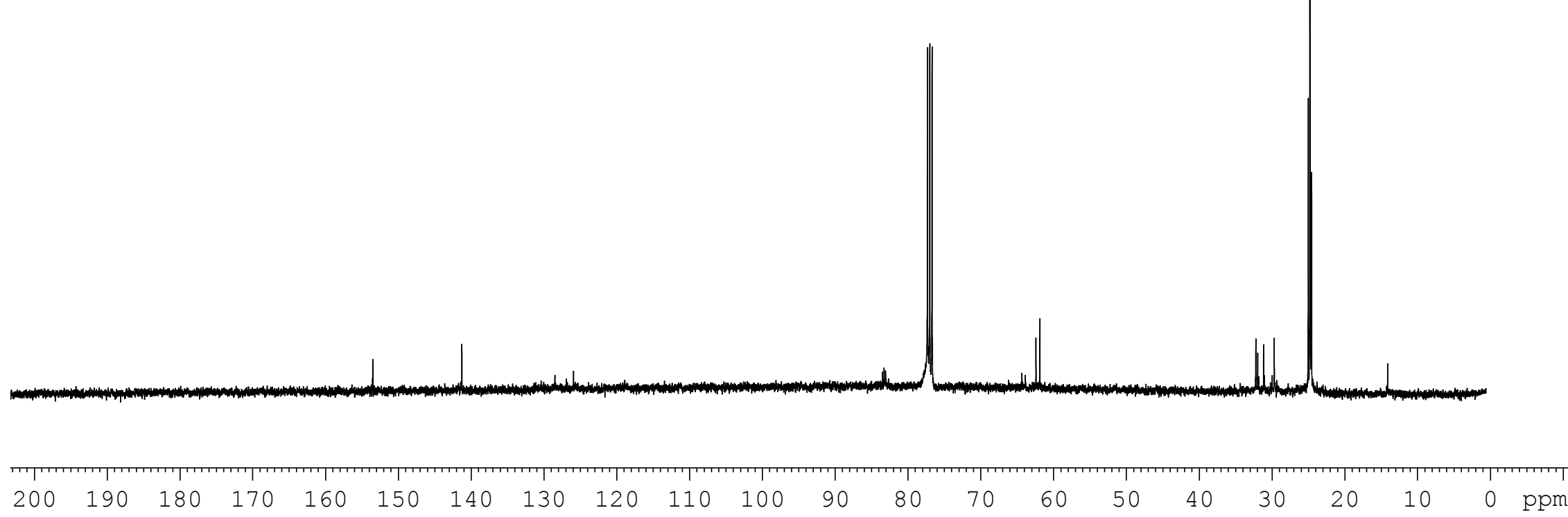

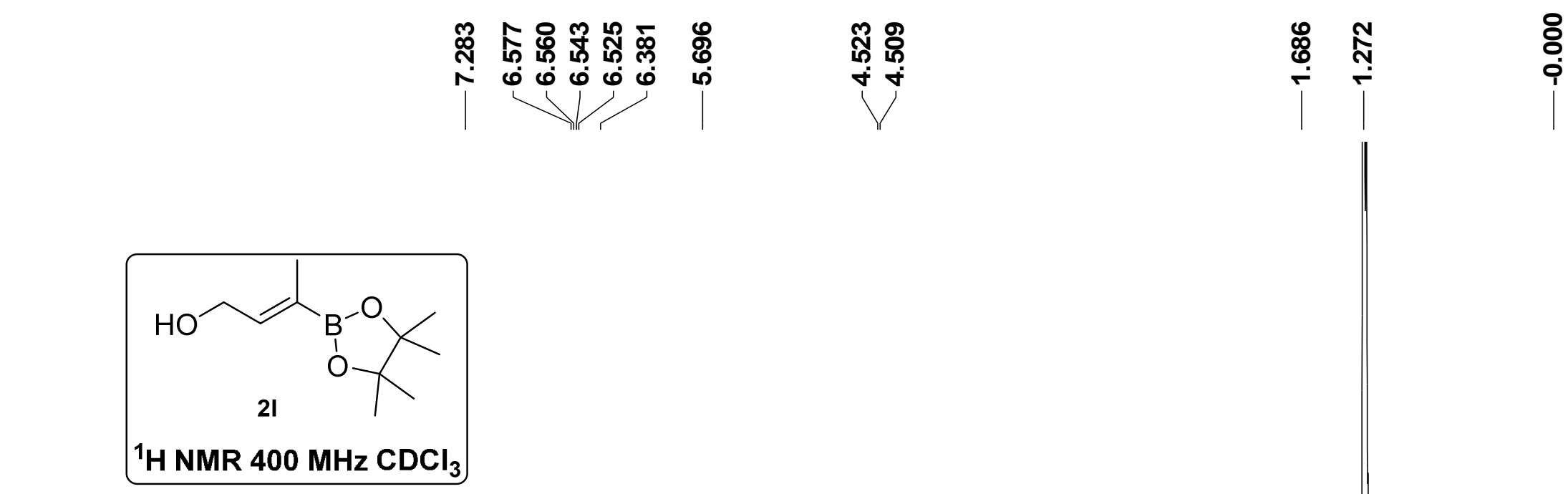

i

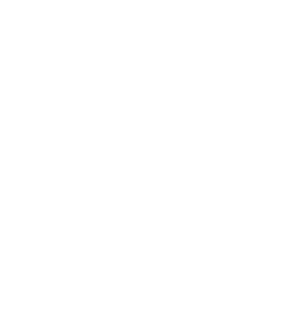




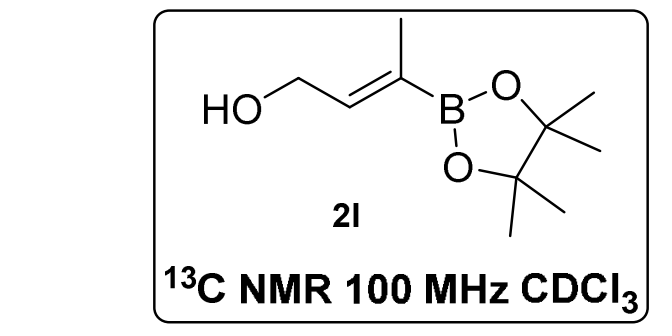

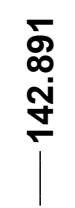

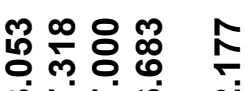

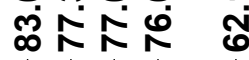

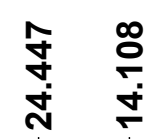
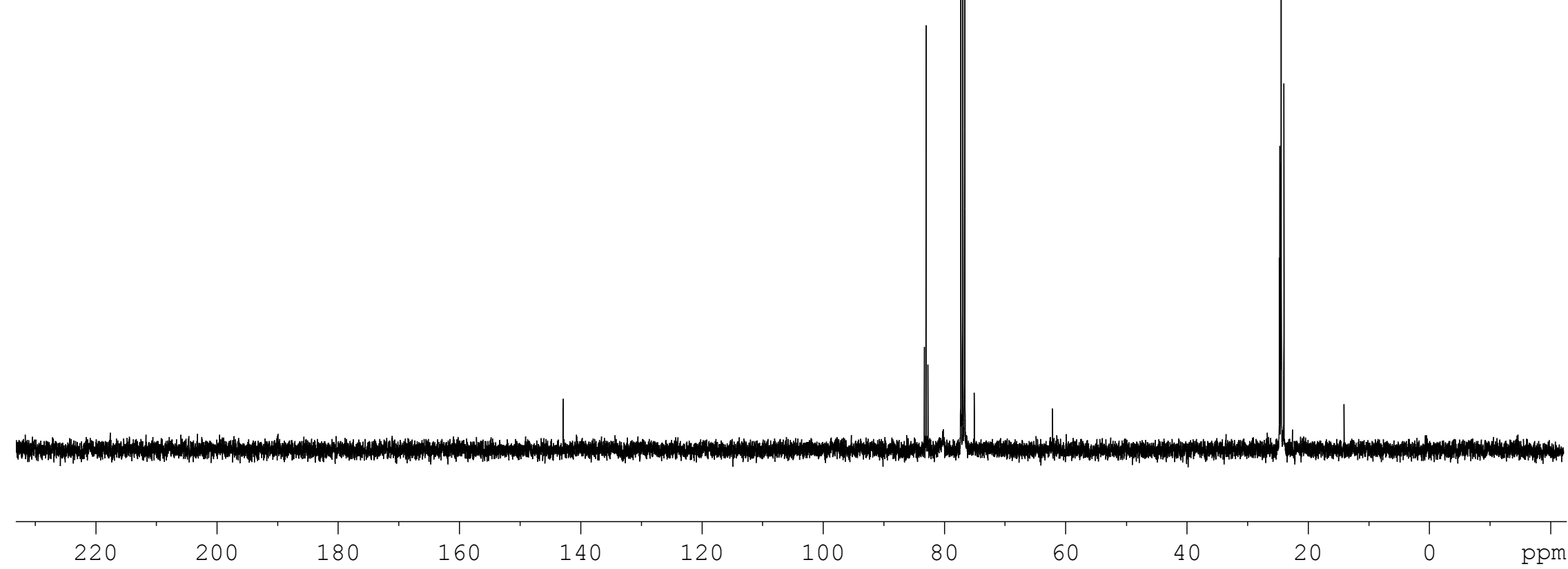


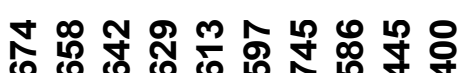
$0.08 .0 \%$

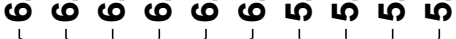
।IIIII

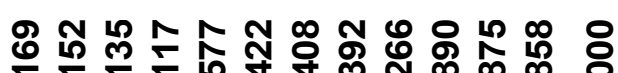

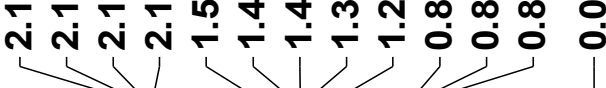
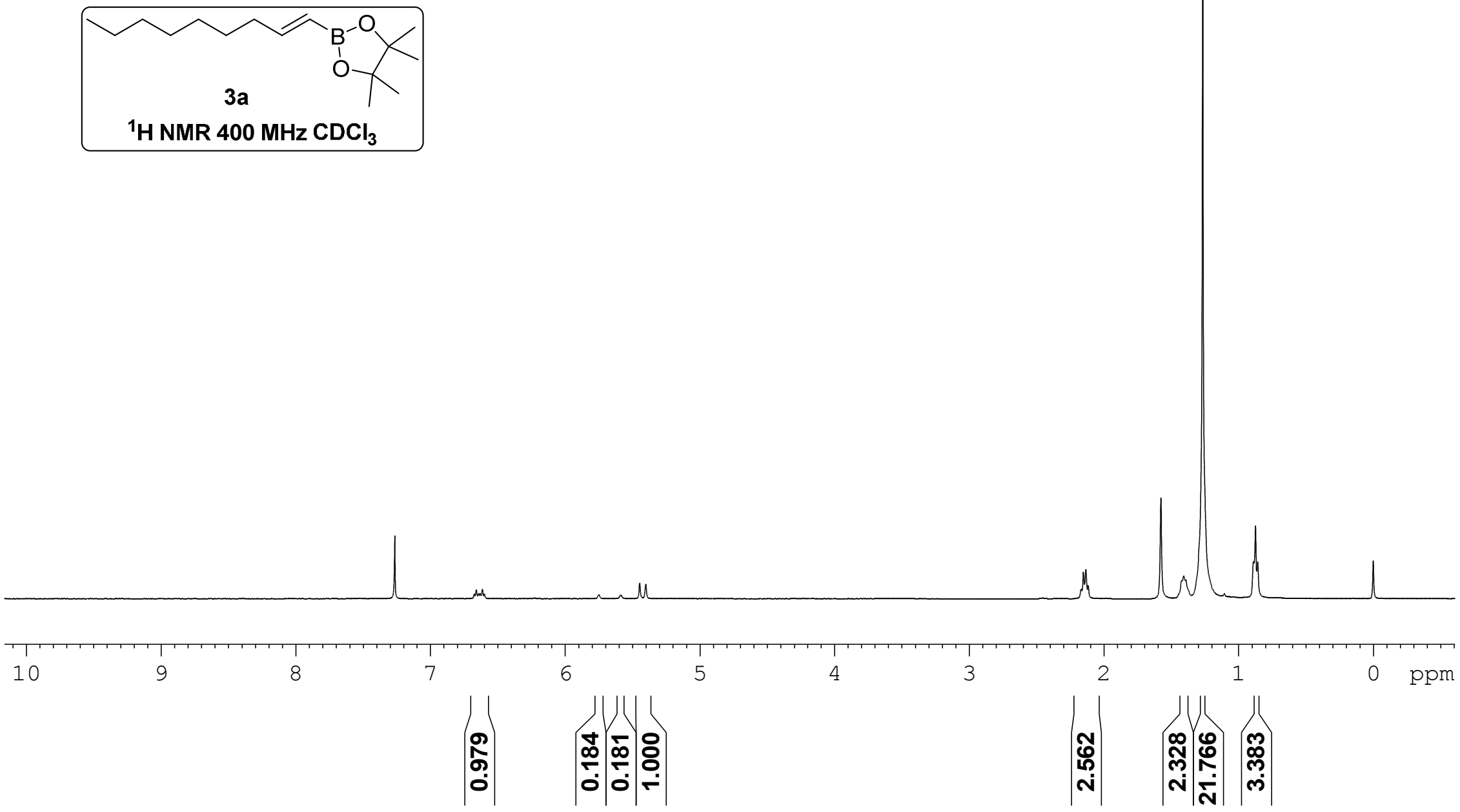


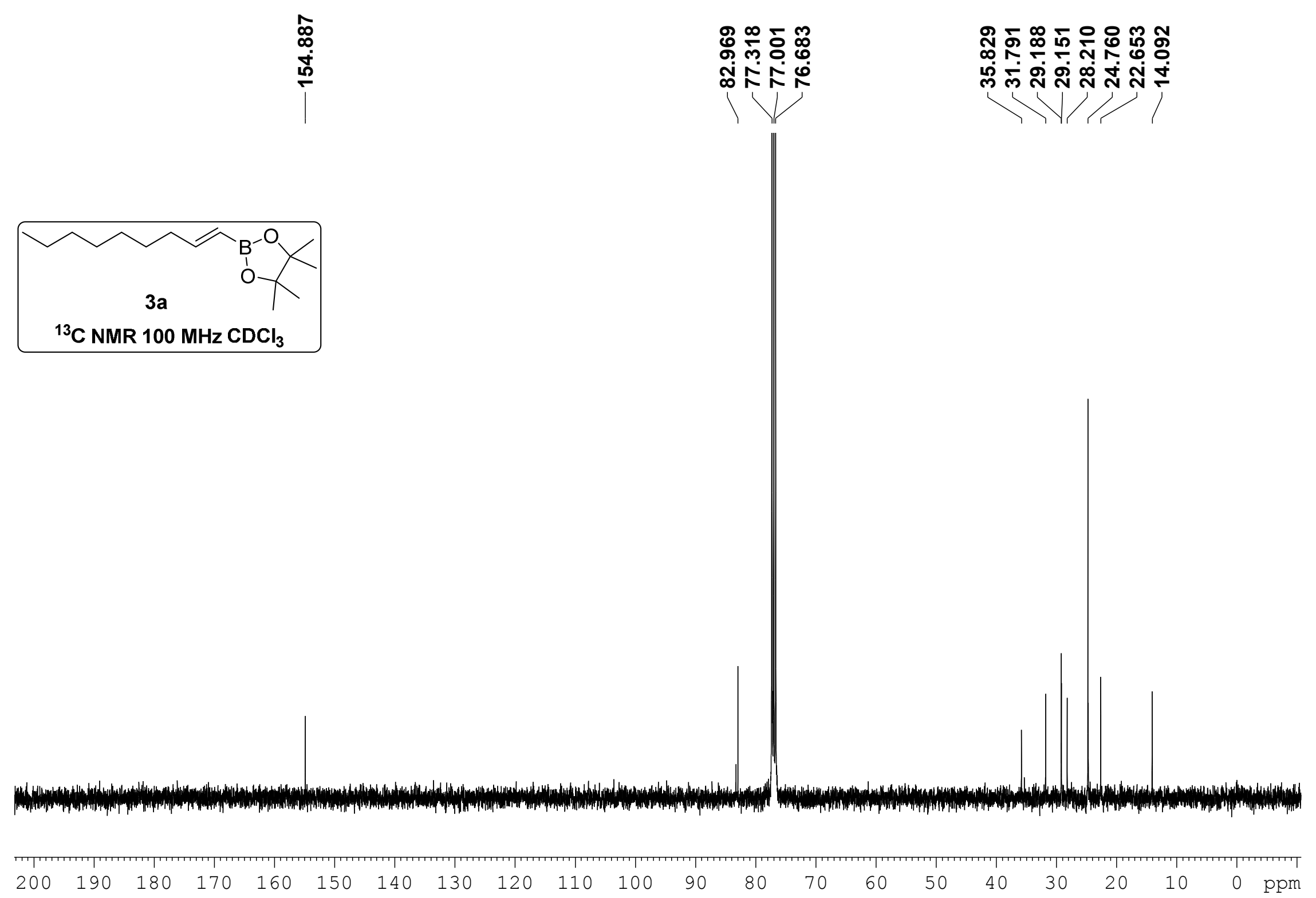




\section{5 ก}

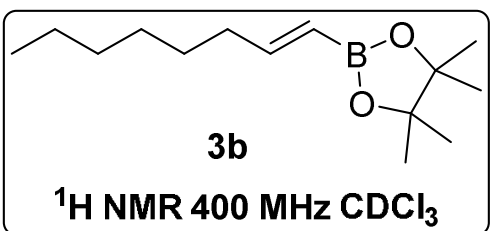

โุ สั่

$>11111$ 
ธำ

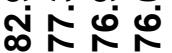

$1 V$
గొ

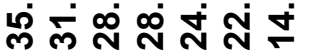

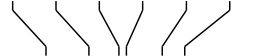

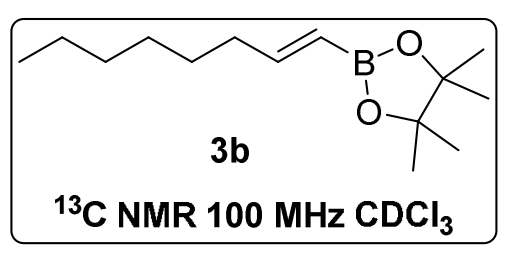
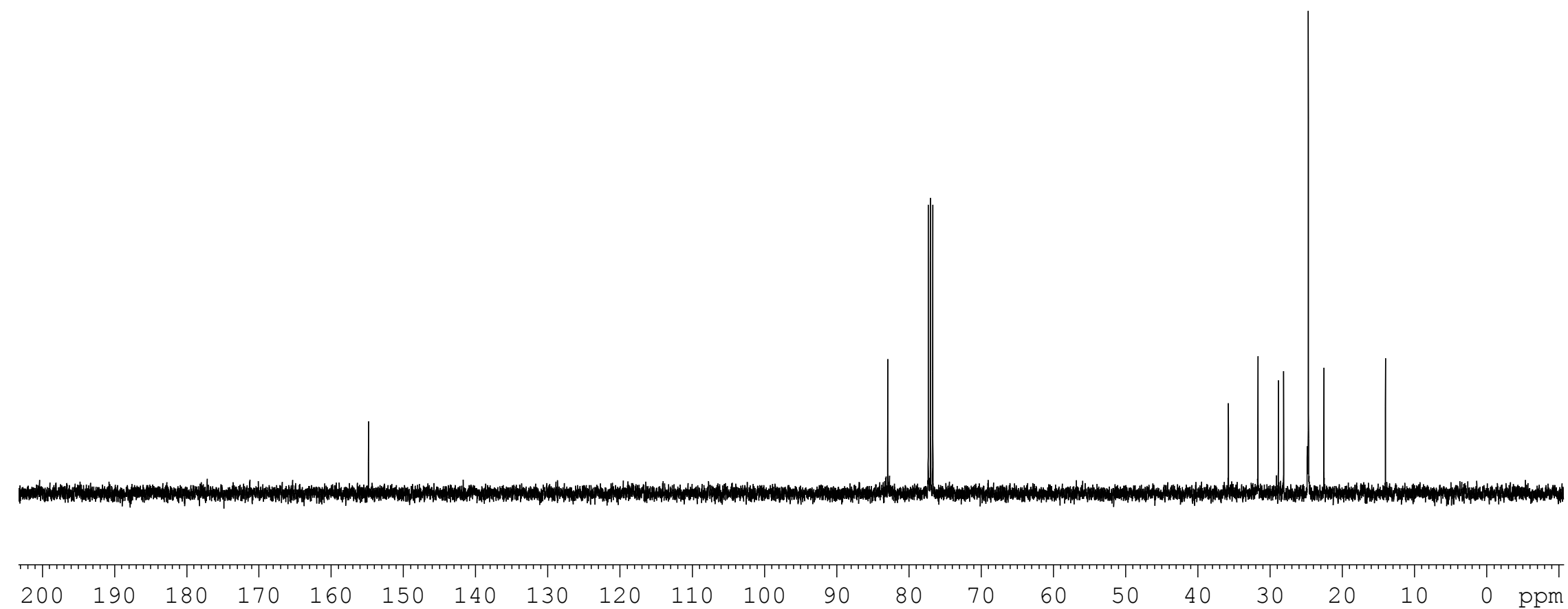

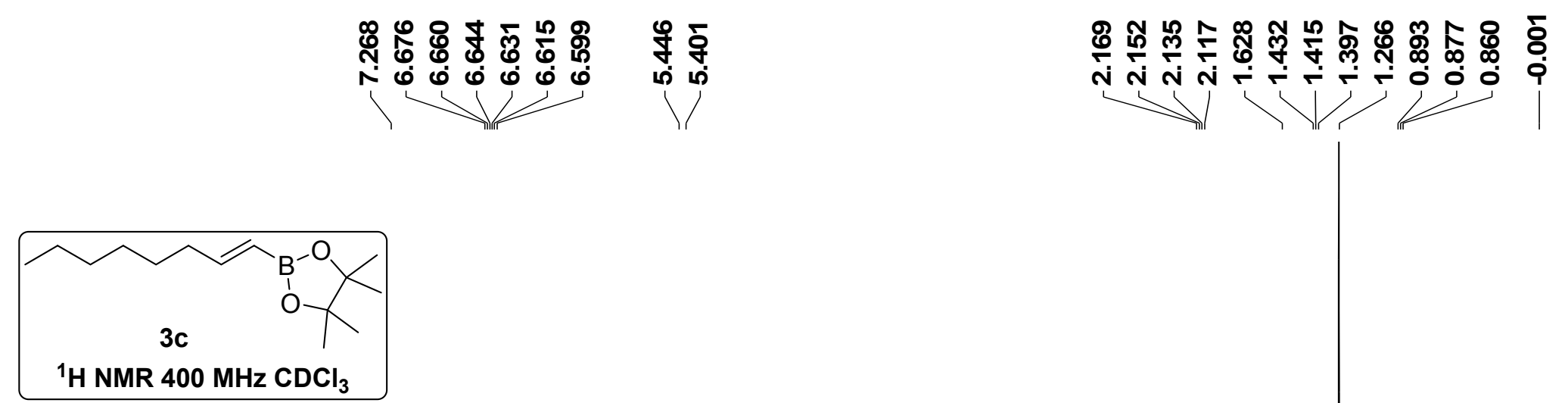

$400 \mathrm{MHz}^{\mathrm{CDCl}_{3}}$

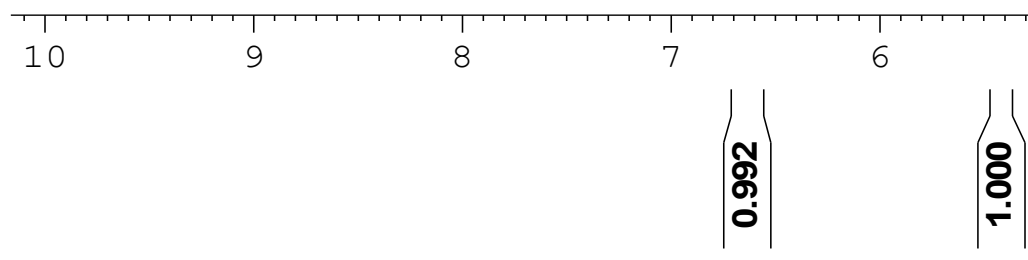




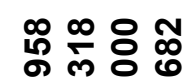

๙

$1 V$

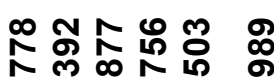

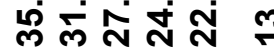

1111

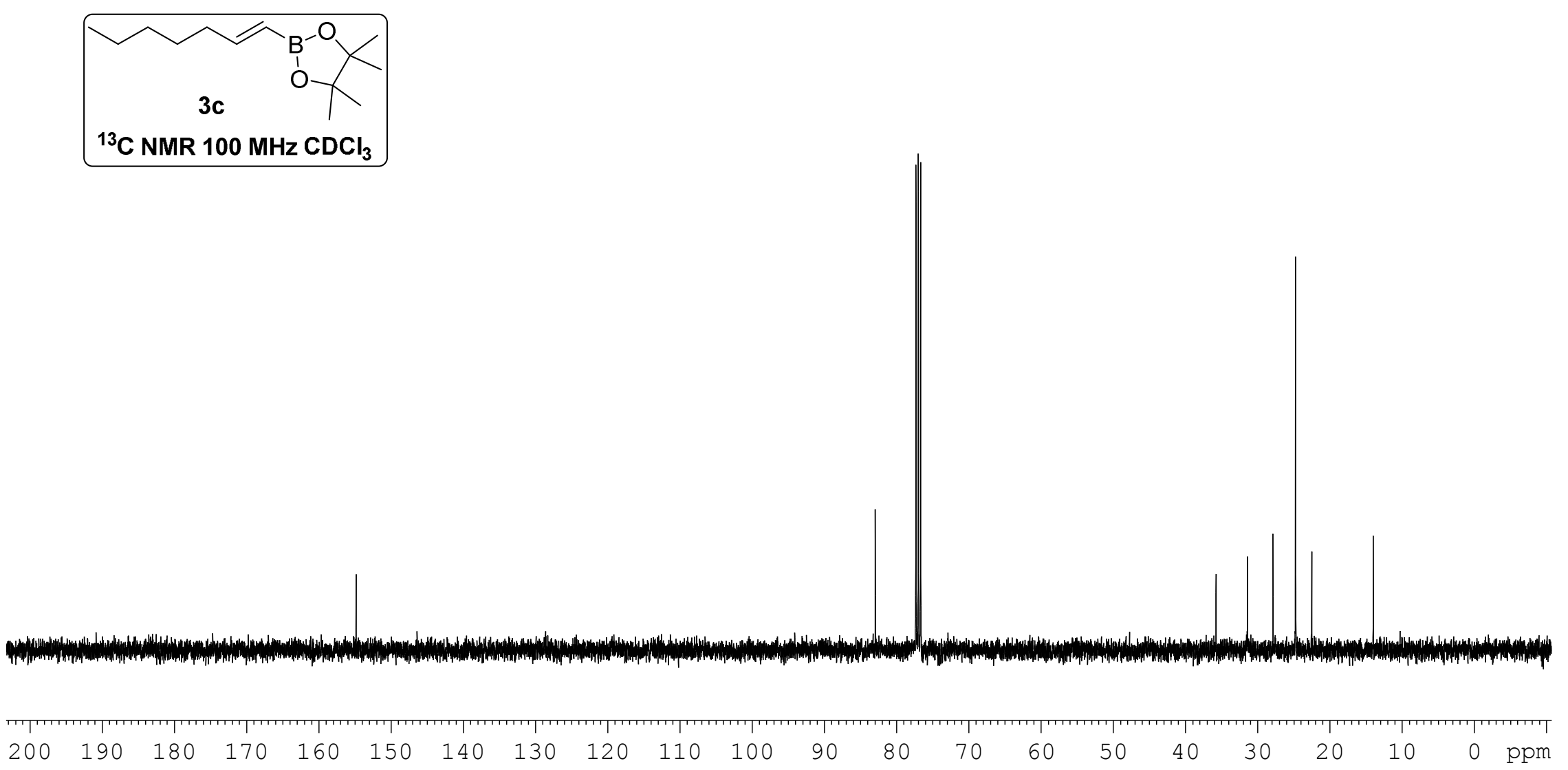



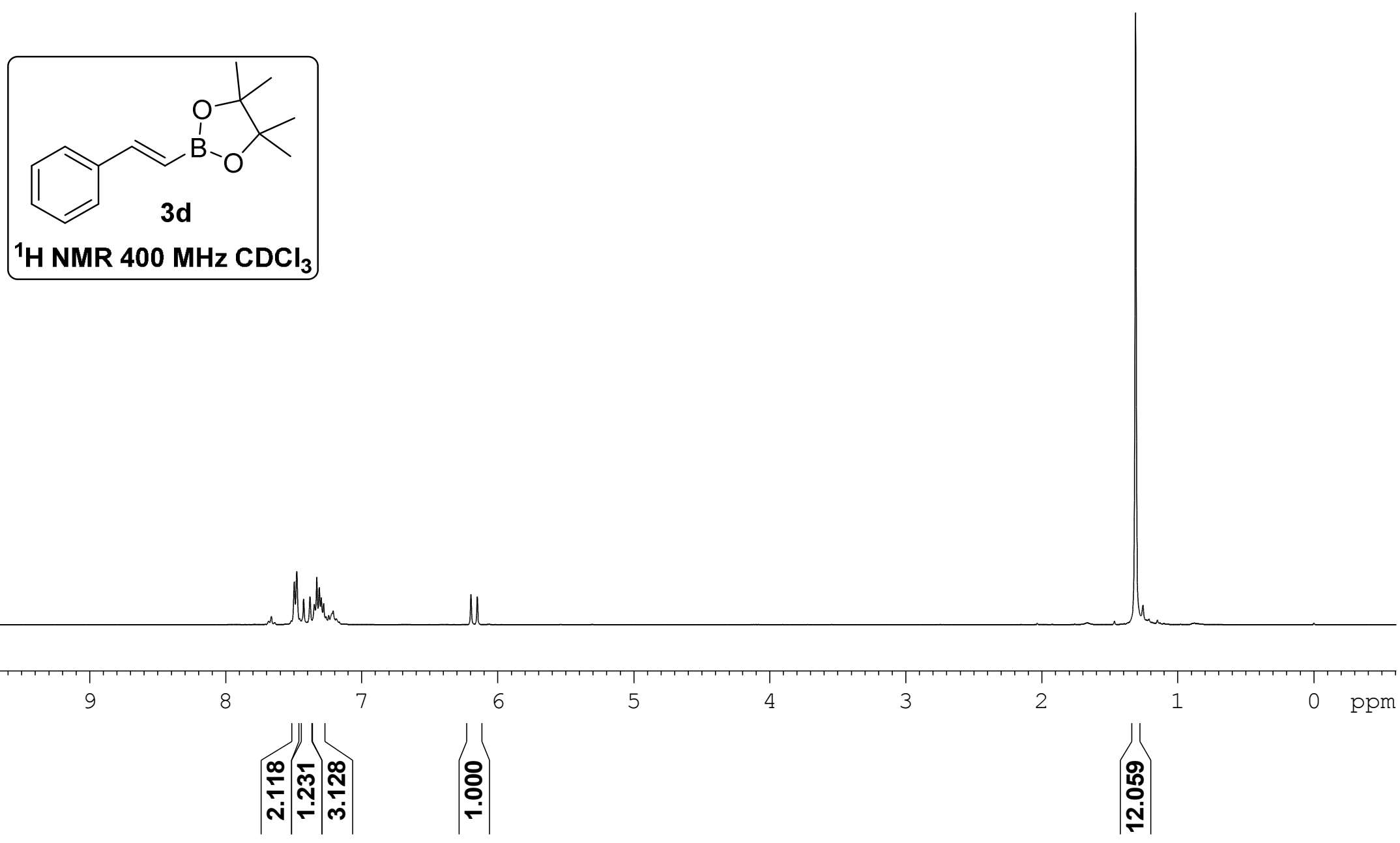


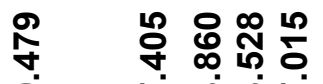

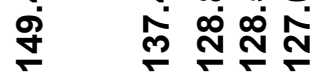

串 웅

のヘト゚

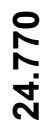

$1 V$

I

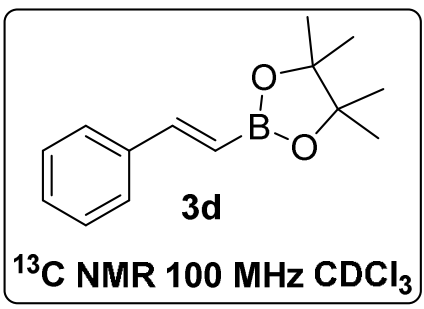

${ }^{13} \mathrm{C} \mathrm{NMR} 100 \mathrm{MHz}^{\mathrm{CDCl}}{ }_{3}$
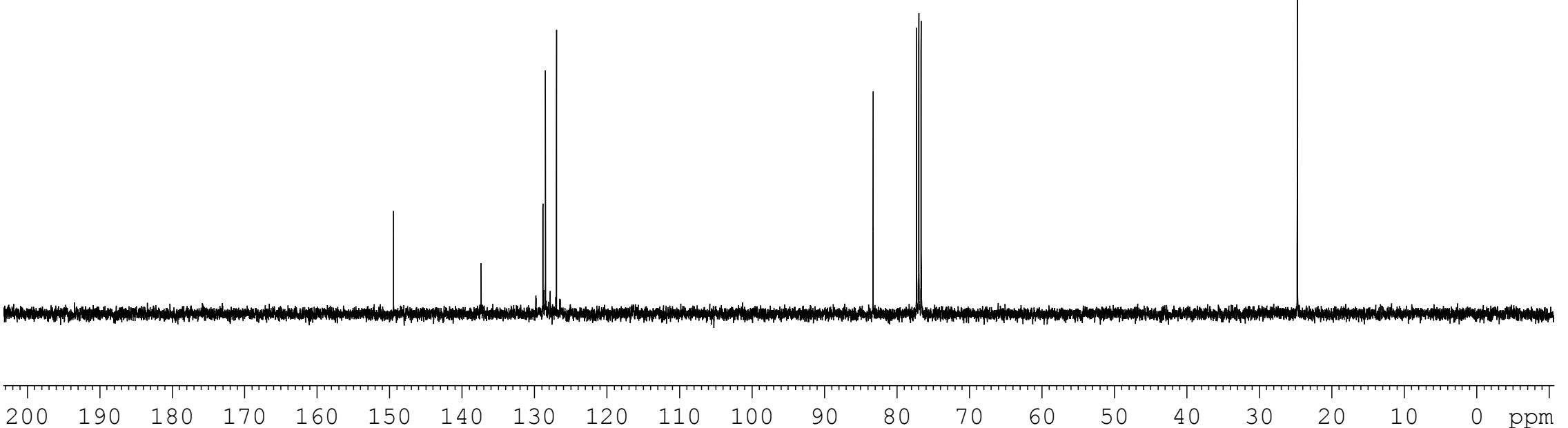

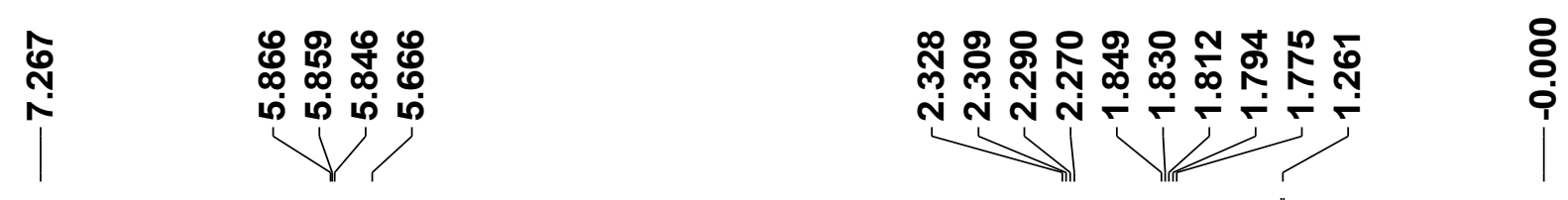

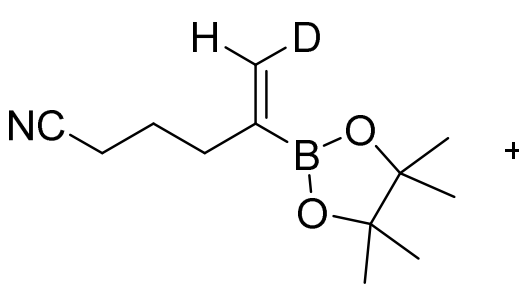

$39 \%$

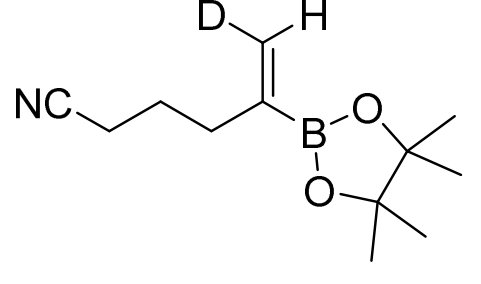

$52 \%$

${ }^{1} \mathrm{H}$ NMR $400 \mathrm{MHz}^{\mathrm{CDCl}}{ }_{3}$
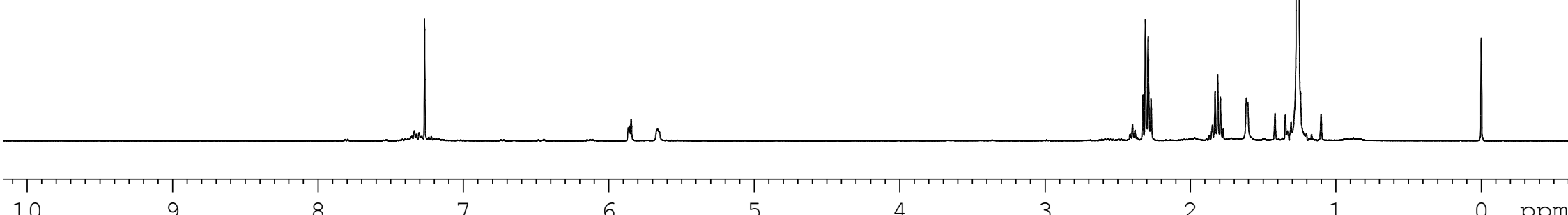

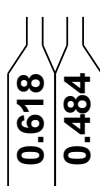

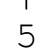

1

0 ppm

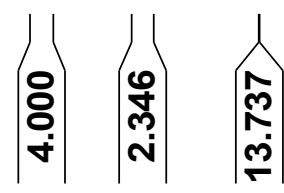




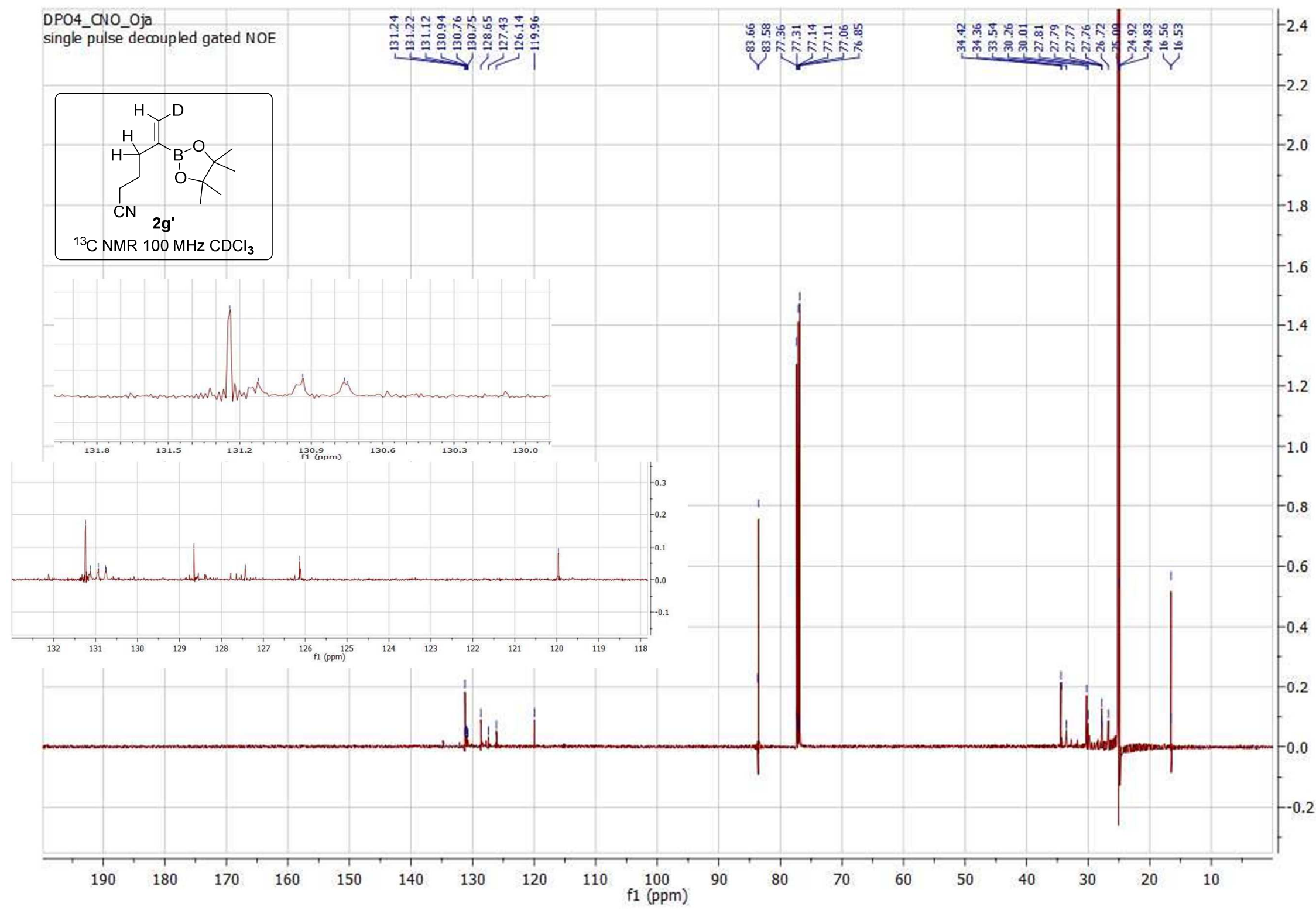


芦
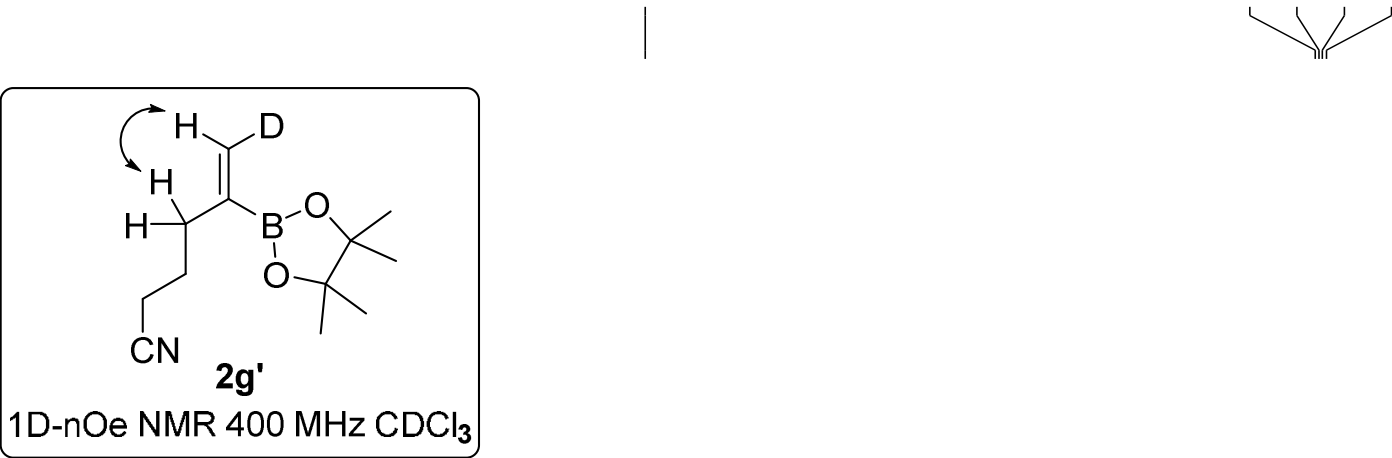

m.

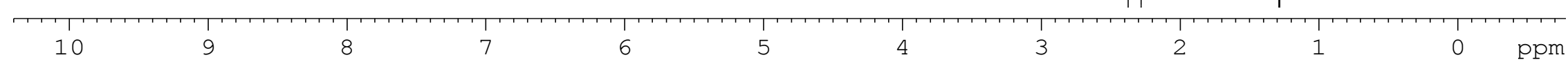




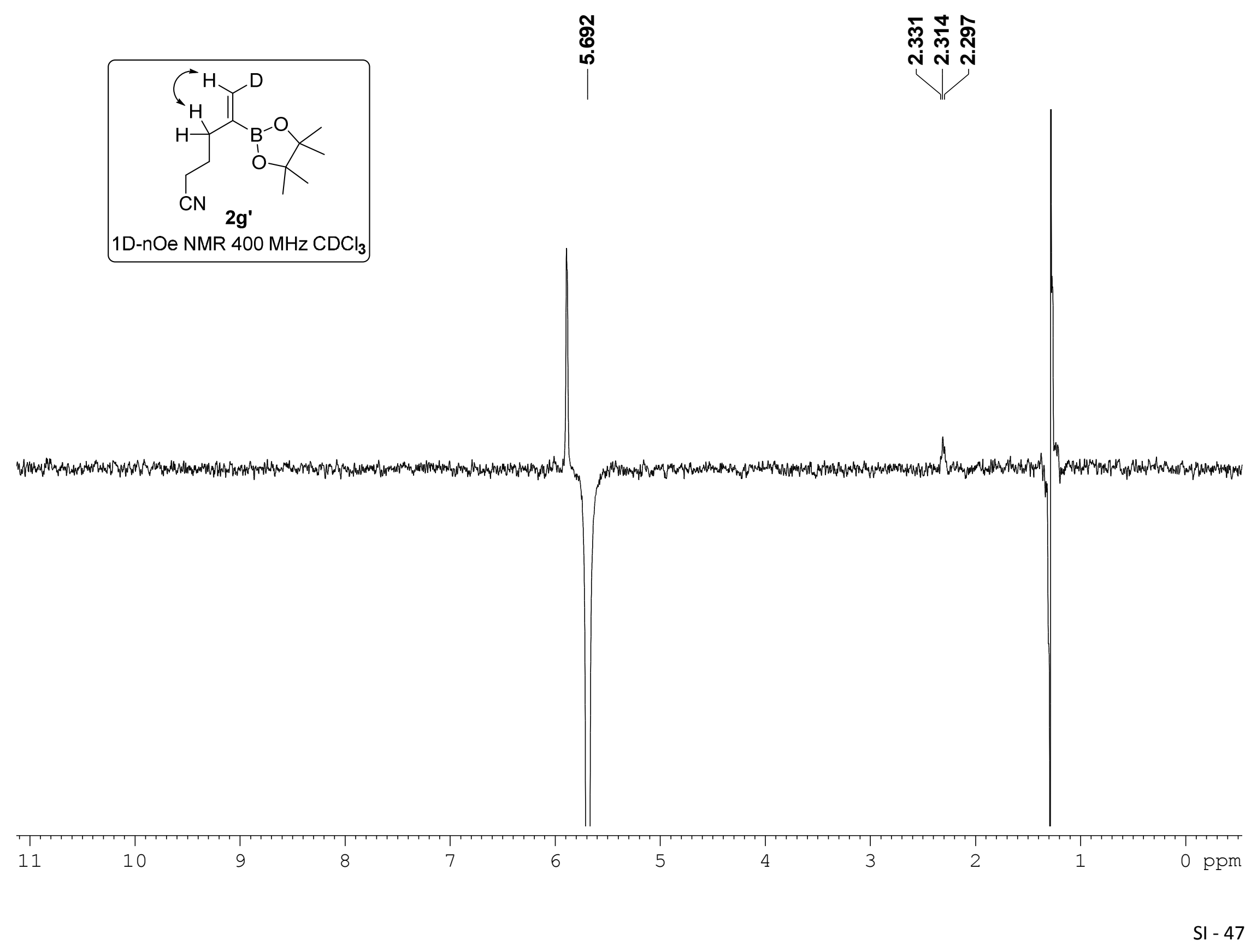


œ

ம்

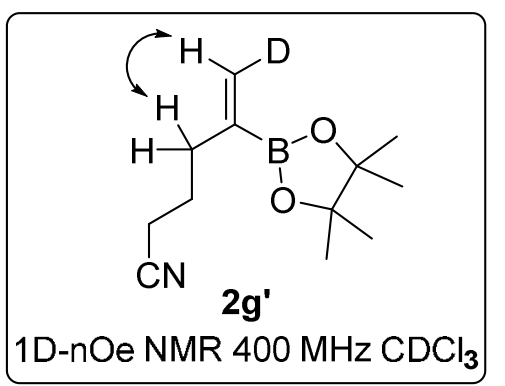



1D-nOe NMR $400 \mathrm{MHz} \mathrm{CDCl}_{3}$

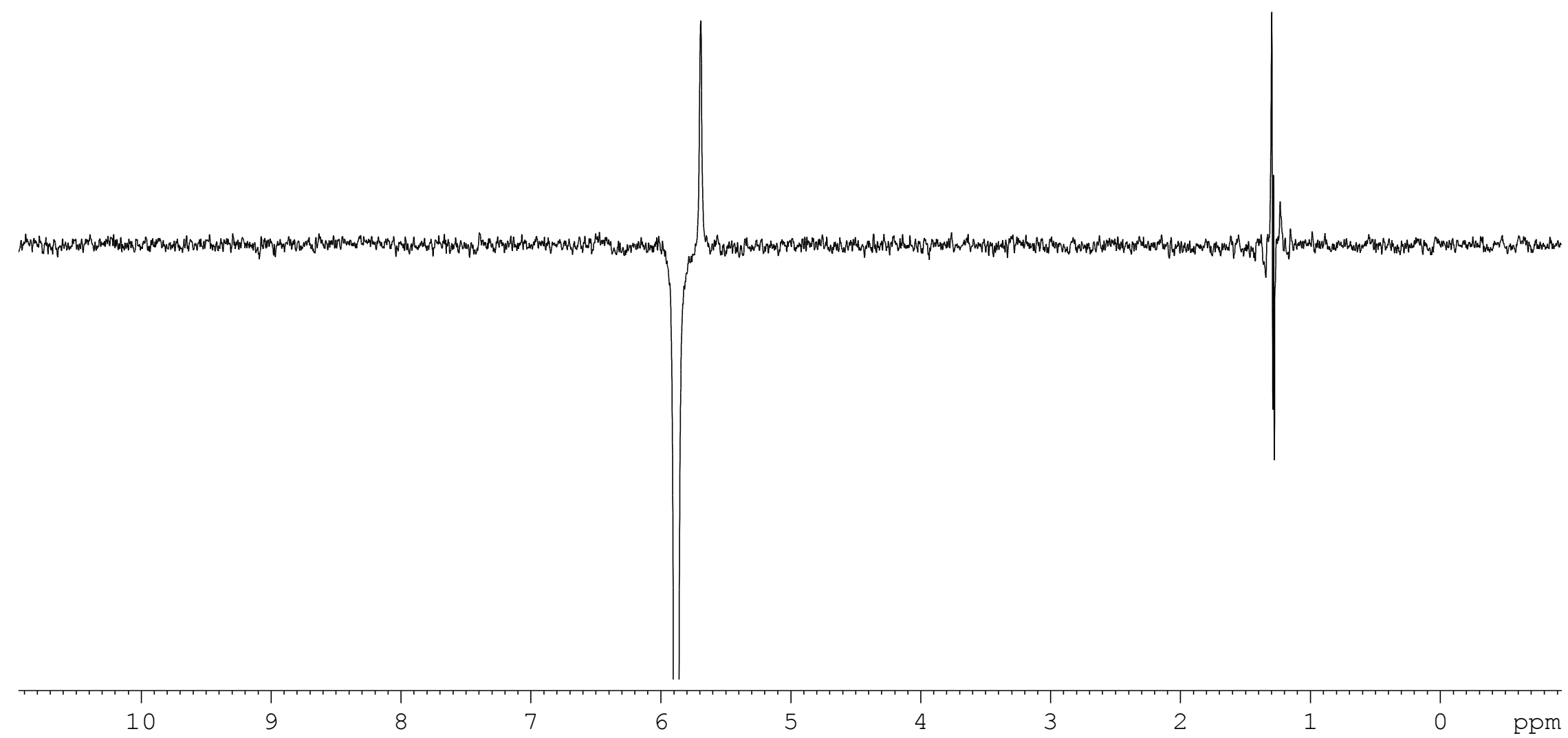




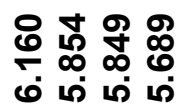

ợ

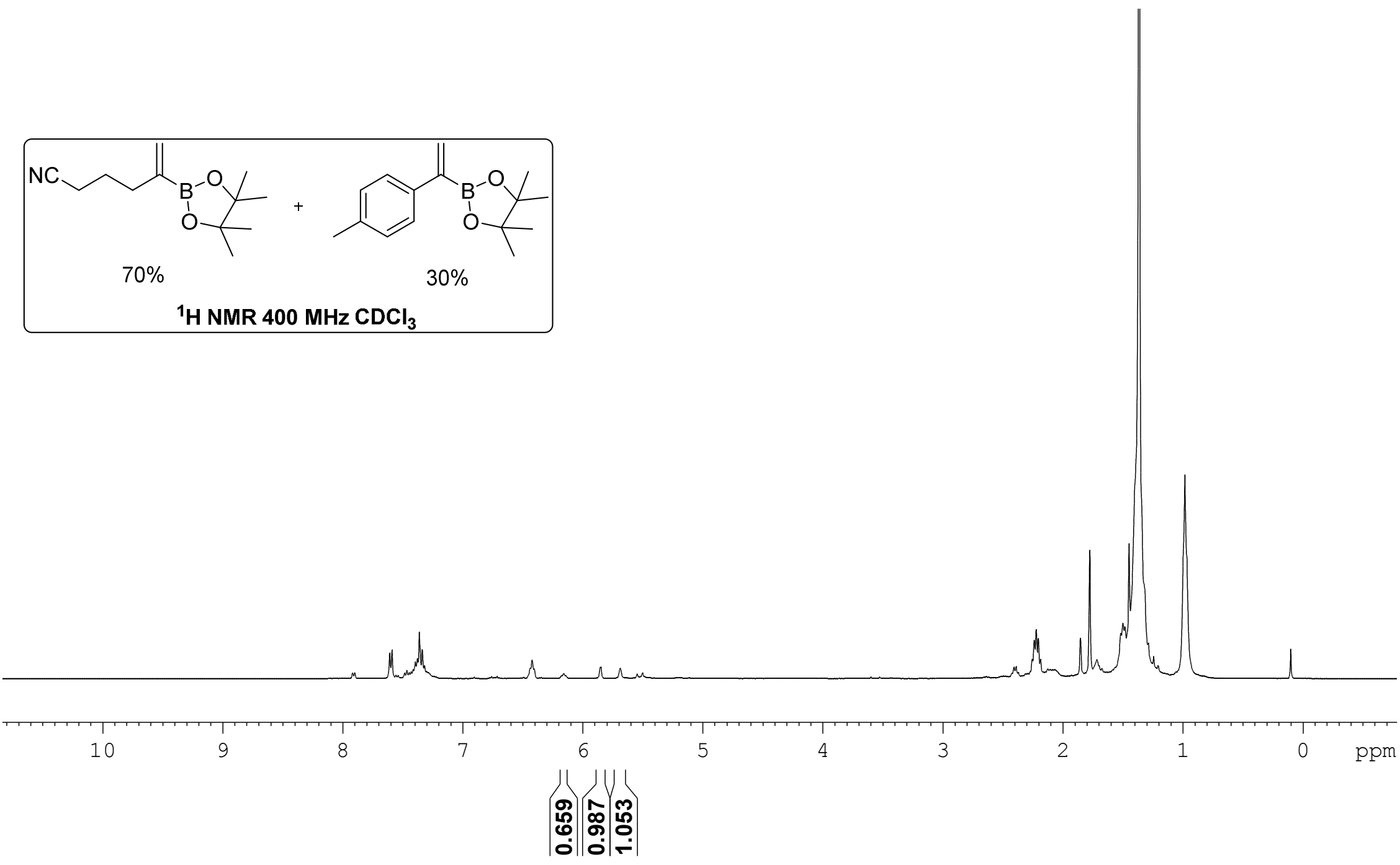

V। 